\title{
Optimal DePloyment of Charging LANES For Electric Vehicles in TRANSPORTATION NETWORKS
}

\author{
Zhibin Chen ${ }^{\mathrm{a}}$, Fang $\mathrm{He}^{\mathrm{b}}$ and Yafeng Yin ${ }^{\mathrm{a}, *}$ \\ ${ }^{a}$ Department of Civil and Coastal Engineering, University of Florida, 365 Weil Hall, Gainesville, \\ FL 32611-6580, United States \\ ${ }^{b}$ Department of Industrial Engineering, Tsinghua University, N502 Shunde Building, \\ Beijing, 100084, P.R. China
}

\begin{abstract}
Given the rapid development of charging-while-driving technology, we envision that charging lanes for electric vehicles can be deployed in regional or even urban road networks in the future and thus attempt to optimize their deployment in this paper. We first develop a new user equilibrium model to describe the equilibrium flow distribution across a road network where charging lanes are deployed. Drivers of electric vehicles, when traveling between their origins and destinations, are assumed to select routes and decide battery recharging plans to minimize their trip times while ensuring to complete their trips without running out of charge. The battery recharging plan will dictate which charging lane to use, how long to charge and at what speed to operate an electric vehicle. The speed will affect the amount of energy recharged as well as travel time. With the established user equilibrium conditions, we further formulate the deployment of charging lanes as a mathematical program with complementarity constraints. Both the network equilibrium and design models are solved by effective solution algorithms and demonstrated with numerical examples.
\end{abstract}

Keywords: electric vehicles, charging-while-driving, charging lane, equilibrium, deployment plan, social cost

\section{Introduction}

Owing to the rapid development of battery technology and growing concern about greenhouse effect, the adoption of electric vehicles (EVs) has grown significantly in the past few years. For example, EV sales in the U.S. have increased to approximately 120,000 in 2014, about six times of the sales in 2011 (Geier, 2015). Although they only made up about $0.73 \%$ of total vehicle sales, some have predicted that their market share would reach $50 \%$ by 2030 (EPRI, 2007). It is

* Corresponding author. Tel.: 352-392-9537 Ext. 1455; Email: yafeng@ufl.edu 
well recognized that the deployment of public charging infrastructure plays a critical role in nurturing the EV market and promoting the adoption of EVs (e.g., Morrow et al., 2008; He et al., 2013a; Nie and Ghamami, 2013). Among various types of charging technologies, chargingwhile-driving (CWD) holds great promise. It can be achieved by either conductive or inductive charging. The former is similar to the technology used for trams and trains, charging EVs via lines overhead or metal bars in the pavement, while the latter, often referred to as dynamic wireless charging, transmits power without using any physical connector; the enabling mechanism includes, among others, inductive coupling, magnetic resonance coupling and microwaves (Vilathgamuwa and Sampath, 2015). CWD can electrify roads to be a charging infrastructure (He et al., 2013b). With charging lanes deployed, EV drivers may not fear any more running out of battery en route. Such a pervasive wireless charging platform can mitigate or even eliminate the "range anxiety" of EV drivers and further boost the adoption of EVs.

Recent progress shows CWD and charging lanes are on the horizon (e.g., Cirimele et al., 2014; Lukic and Pantic, 2013; Ning et al., 2013; Yu et al., 2011; Choi et al., 2013; Shinohara et al., 2013; Vilathgamuwa and Sampath, 2015; Fuller, 2016). The Energy Dynamics Laboratory at Utah State University has proved that enough power can be transferred wirelessly to safely and effectively charge electric vehicles (Morris, 2015). Future versions of their system are expected to wirelessly charge vehicles at a speed of $75 \mathrm{mph}$. Companies such as Scania and Qualcomm are also developing their own inductive charging technologies. 15 miles of charging lanes have been constructed in Gumi, South Korea, which recharge a dozen of buses while in motion (Bansal, 2015). Scania and Siemens are investigating an overhead charging technology and have a two-kilometer test track outside Berlin (Scania Newsroom, 2014; Herron, 2014). Volvo is field testing two conductive charging technologies at a 400-meter track near Gothenburg, Sweden (Schiller, 2013). The British government is working with BMW, Renault and Scania to test dynamic wireless charging technology in 2015 (TRL, 2015). Siemens, in conjunction with Volvo, is to trial an eHighway system on a two-mile stretch of highway in California in the vicinity of the ports of Los Angeles and Long Beach (Siemens, 2014). Recently, after completing a feasibility study, Highways England has announced to trial charging lanes in 2015 (Highways England, 2015a,b).

We thus envision in this paper that charging lanes are deployed in general road networks via either conductive or inductive charging. With charging lanes deployed, drivers of EVs, when traveling between their origins and destinations, can select routes and decide battery recharging plans to minimize their trip times while preventing the batteries of their vehicles from running out of charge. A battery recharging plan will dictate which charging lane to use, how long to charge, and at what speed to operate an EV. The operating speed will decide how long the EV will stay on a charging lane, thereby affecting the amount of electricity that can be recharged. More specifically, a lower operating speed will allow more time to recharge but yield higher travel time. Therefore, EV drivers need to make a tradeoff between their energy need and travel time. Although previous studies have formulated network equilibrium models with EVs (e.g., Jiang et al., 2012, 2014; He et al., 2014, 2015, 2016), all focus on accommodating the limited driving range of EVs, and none is able to capture such a tradeoff, which arises uniquely in the context of charging lanes. With the tradeoff, the relationship between traffic volume and actual 
travel time is no longer described by a single link performance function. Drivers' choice of operating speeds on charging links need to be explicitly modeled. In this paper, we will first consider EV drivers' route choice and recharging decision over a network where charging lanes are deployed and formulate a new user equilibrium model to describe the consequent equilibrium conditions.

We then proceed to optimize the deployment plan of charging lanes over a general network. A large body of literature has been devoted to optimal locations of refueling, recharging or battery swapping stations for EVs in a metropolitan area (see, e.g., Dong et al., 2014; Jung et al., 2014; Riemann et al., 2015). However, most of them do not consider the impacts of charging infrastructure on EV drivers' travel choices. Among a few exceptions, He et al. (2013a) assumed that the availability of charging opportunities may affect drivers' destination choices and subsequently allocate charging stations to maximize social welfare associated with transportation and power networks. Kang and Recker (2014) investigated individuals' activity scheduling and routing with refueling, and then determined optimal locations of refueling stations for alternative fuel vehicles. Jung et al. (2014) proposed a bi-level programing model for locating electric taxi charging stations where a dispatch algorithm at the lower level determines where a taxi should be replenished and which station the taxi should visit. Recently, He et al. (2015) optimized the location of charging stations by considering EV drivers' adjustments of travel and recharging decisions. In this paper, as charging lanes will inevitably affect drivers' route choice and recharging decision, such spontaneous reactions need to be proactively accommodated when optimizing the locations of charging lanes.

For the remainder, Section 2 presents the operational concept of charging lanes considered in this paper and basic assumptions for the proposed models. Section 3 formulates a network equilibrium model and discusses its solution algorithm. The deployment of charging lanes is optimized in Section 4. Lastly, Section 5 concludes the paper.

\section{Basic Considerations}

It is envisioned that a government agency strategically locates charging lanes along certain road segments. When traveling between their origins and destinations, drivers of EVs make route choices and decide whether to use the lanes. They can decide where to enter and leave the lanes, how long to charge, and at what speeds they operate their vehicles. It is plausible that the amount of energy transferred from a charging lane to an EV will depend on the time that the vehicle stays on the lane. Therefore, driving speed is one of the critical decisions for charging an EV. Drivers can also decide whether to charge their vehicles while driving on charging lanes. In other words, EVs are not necessarily charged on charging lanes.

Since the overarching goal of this paper is to optimize the deployment plan of charging lanes, a static game-theoretic modeling framework is adopted, which does not allow us to fully capture the operational characteristics of charging lanes. Below we summarize our basic considerations or assumptions for the modeling and analysis of charging lanes in this paper: 
i. A link in the network is either a regular or charging link. All lanes on a charging link are charging lanes. This consideration is not overly restrictive as we can always represent charging lanes as separate links.

ii. All vehicles in the network are EVs with the same battery size and initial state of charge (SOC). This assumption can be easily relaxed by introducing multiple classes of EVs.

iii. Compared with the travel-time cost, the electricity cost is insignificant (He et al., 2014). Therefore, EV drivers are assumed to select routes to minimize their travel time cost while ensuring not to run out of charge before reaching their destinations.

iv. An EV driver can decide whether to charge his or her vehicle when driving on a charging link. If he or she decides to charge, the amount of electricity charged is equal to a constant charging rate multiplying the charging time. Apparently, charging time is no longer than the time that the vehicle spends on traversing a charging link.

v. On a charging link, an EV driver can choose his or her travelling speed between a maximum currently allowable speed and a minimum speed limit. The former can be specified by the link performance function based on the flow of the link (Yang et al., 2015), while the latter is a predetermined constant and is assumed to be no greater than the former. When a fast-travelling vehicle catches up a slow-moving one, it is always possible for the vehicle to perform overtaking maneuvers so that its speed choice is not affected. The maximum currently allowable speed is thus assumed to only depend on the flow of the link.

vi. The energy consumption of an EV is proportional to the distance that it has travelled. This assumption is particularly valid when the travel speed of the EV is less than $65 \mathrm{mph}$ as per previous studies such as Wu et al. (2015). We note that some other studies, such as Fontana (2013), suggested that the energy consumption of an EV is not necessarily proportional to the distance travelled, but depends on its velocity and acceleration/deceleration etc. We further note that advancements in electric drive system may further change the energy consumption profile of an EV. Therefore, this assumption can be strong in many contexts and more realistic energy consumption models need to be used instead. However, as relaxing this assumption while capturing speed selections on charging lanes appears to be mathematically intractable, we leave it to our future study.

For the convenience of readers, below we list some notations frequently used in the paper. 


\begin{tabular}{|c|c|}
\hline \multicolumn{2}{|l|}{ Sets } \\
\hline$N$ & Set of nodes \\
\hline$A$ & Set of links \\
\hline$\hat{A}$ & Set of charging links \\
\hline$W$ & Set of O-D pairs \\
\hline$P^{w}$ & Set of paths between O-D pair $w \in W$ \\
\hline$\hat{P}^{w}$ & Set of usable paths between O-D pair $w \in W$ \\
\hline$N(p)$ & Set of nodes of path $p \in P^{w}$ between O-D pair $w \in W$ \\
\hline$A(p)$ & Set of links along path $p \in P^{w}$ between O-D pair $w \in W$ \\
\hline$\hat{A}(p)$ & Set of charging links along path $p \in P^{w}$ between O-D pair $w \in W$ \\
\hline \multicolumn{2}{|c|}{ Parameters } \\
\hline$a$ & Link $a=(i, j) \in A$ \\
\hline$w$ & O-D pair $w \in W$ \\
\hline$g^{w}$ & Travel demand between O-D pair $w \in W$ \\
\hline$p$ & Path $p \in P^{w}$ between O-D pair $w \in W$ \\
\hline$o(w)$ & Origin of O-D pair $w \in W$ \\
\hline$d(w)$ & Destination of O-D pair $w \in W$ \\
\hline$d_{a}$ & Distance of link $a \in A$ \\
\hline $\bar{t}_{a}$ & Travel time of link $a \in \hat{A}$ when travelling with the minimum speed limit \\
\hline$L_{0}$ & Initial SOC \\
\hline$L_{\max }$ & Battery size \\
\hline$\varpi$ & Energy consumption rate of electric vehicles \\
\hline$e$ & Recharging rate of charging lanes \\
\hline$m^{w}$ & $\begin{array}{l}\text { Minimum SOC that a driver will feel comfortable with when operating the EV } \\
\text { between O-D pair } w \in W\end{array}$ \\
\hline$I$ & Budget \\
\hline$c_{a}$ & Cost for constructing one mile of charging lanes on link $a \in A$ \\
\hline \multicolumn{2}{|c|}{ Variables } \\
\hline$f_{p}$ & Traffic flow on path $p \in P^{w}$ between O-D pair $w \in W$ \\
\hline$v_{a}$ & Traffic flow of link $a \in A$ \\
\hline$t_{a}\left(v_{a}\right)$ & Travel time of link $a \in A$ specified by the performance function of the link \\
\hline$s_{a}^{p}$ & $\begin{array}{l}\text { Actual travel time that the vehicle spends on charging link } a \in \hat{A} \text { along path } \\
p \in P^{w} \text { between O-D pair } w \in W\end{array}$ \\
\hline$\hat{s}_{a}^{p}$ & $\begin{array}{l}\text { Recharging time on charging link } a \in \hat{A} \text { along path } p \in P^{w} \text { between O-D pair } \\
w \in W\end{array}$ \\
\hline$L_{i}^{p}$ & SOC at node $i \in N$ along path $p \in P^{w}$ between O-D pair $w \in W$ \\
\hline$y_{a}$ & $\begin{array}{l}\text { A binary variable, representing whether link } a \text { should be converted into a } \\
\text { charging link. If yes, } y_{a}=1 \text {; otherwise, } y_{a}=0 \text {. }\end{array}$ \\
\hline
\end{tabular}




\section{Network Equilibrium Model}

Let $G(N, A)$ denote the network where $N$ and $A$ are the sets of nodes and links in the network respectively. Further, $\hat{A}$ is the set of charging links. We represent a link as $a \in A$ or its starting and ending nodes, i.e., $a=(i, j) \in A$. Let $W$ denote the set of origin-destination (O-D) pairs, and let $g^{w}$ and $P^{w}$ represent the travel demand and the set of paths between O-D pair $w \in W$ respectively. Further, we use $f_{p}$ to denote the traffic flow on path $p \in P^{w}$ between O-D pair $w \in W$, and $o(w)$ and $d(w)$ for the origin and destination of O-D pair $w \in W$. Let $\delta_{a}^{p}$ represent the path-link incidence, which equals 1 if path $p \in P^{w}$ consists of link $a \in A$ and 0 otherwise. Let $v_{a}$ and $d_{a}$ be the traffic flow and distance of link $a$. Define $t_{a}\left(v_{a}\right)$ as the travel time of link $a$ specified by the performance function of the link. For a charging link, $t_{a}\left(v_{a}\right)$ is corresponding to the maximum currently allowable speed. For convenience, we hereinafter call them as the prevailing travel time or prevailing speed. Note that the prevailing travel time or speed is not the average time or speed of the traffic stream. Moreover, let $\bar{t}_{a}$ be the travel time when travelling with the minimum speed limit. In other words, $\bar{t}_{a}$ is the maximum travel time of the charging link. According to Assumption v, the travel time of an EV on a charging link should vary between $t_{a}\left(v_{a}\right)$ and $\bar{t}_{a}$. We further note that as per Assumption $\mathrm{v}$, the prevailing speed depends only on the flow of the charging lane, because overtaking opportunities are assumed to be always available. However, some may argue if a substantial number of drivers intentionally slow down for charging, the prevailing speed may be brought down. Appendix A presents a recipe how the model proposed in this study can be adjusted to capture such an effect. The key is to add one additional constraint to require the choice of speed can be only higher than the average speed of the traffic stream to a certain percentage.

Not all paths are usable to EVs due to their limited driving range. We thus formally define a usable path as follows.

Definition 1 (He et al., 2014). A path is usable if an EV is able to complete it with or without recharging.

Fig. 1 is a toy network that we use to illustrate the above definition. Suppose that the O-D demand is 5 and the lengths of link 1-2, 2-3, and 3-4 are 30, 6, and 50 miles respectively. Further, their link performance functions are $t_{12}(v)=30+0.2 v, t_{23}(v)=5+0.1 v$, and $t_{34}(v)=40+0.3 v$ min. The minimum speed limit of each link is $30 \mathrm{mph}$. Consider that the battery size of an EV is $24 \mathrm{kWh}$, and its initial SOC is $10 \mathrm{kWh}$. The energy consumption rate is $0.3 \mathrm{kWh} / \mathrm{mi}$ and the recharging rate is $1 \mathrm{kWh} / \mathrm{min}$. For scenario (a) without any charging link, the path is not usable, because its total distance is $86 \mathrm{mi}$, much longer than the driving range provided by the initial SOC, i.e., 10/0.3 = 33.3 mi. For scenario (b), although link 3-4 is a charging link, the EV cannot reach it with the initial SOC and the path is still not usable. For scenario (c), as the minimum speed limit is $30 \mathrm{mph}$, the maximum travel time of charging link 23 is $12 \mathrm{~min}$. Therefore, the electricity charged on the link cannot be greater than $12 \mathrm{kWh}$. As a result, the driving range provided by the initial SOC and the charging lane is $(10+12) / 0.3=$ 
$73.3 \mathrm{mi}$, less than the total distance of $86 \mathrm{mi}$. The path is still not usable in this scenario. For scenario (d), in order to finish the trip without running out of energy, the vehicle needs to recharge $86 \times 0.3-10=15.8 \mathrm{kWh}$ at link $1-2$, which requires a charging time of $15.8 \mathrm{~min}$, less than the maximum travel time, i.e., $30 / 30=1 \mathrm{~h}$. Therefore, the path is usable in this scenario. Further, since the required recharging time is less than the prevailing travel time, i.e., $t_{12}=30+0.2 \times 5=31 \mathrm{~min}$, the vehicle can operate at the prevailing speed on the charging link.

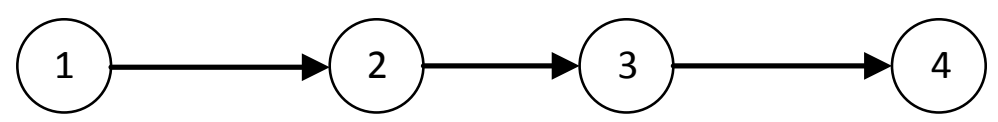

(a)

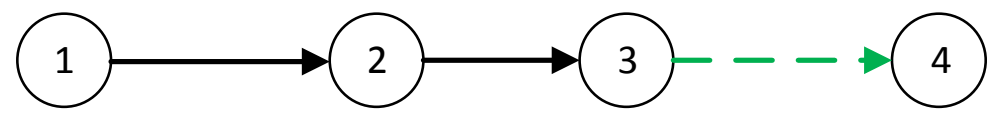

(b)

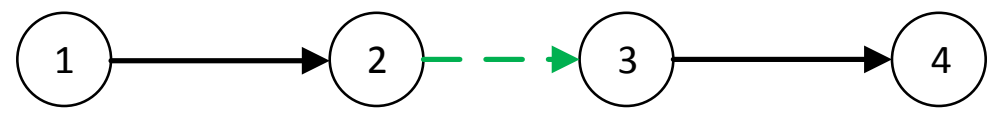

(c)

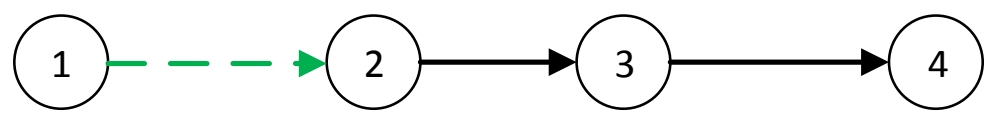

(d)

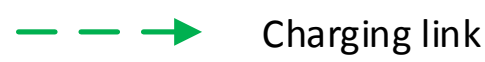

Fig. 1. A toy network with four nodes

Proposition 1. The usability of a path is flow-independent.

Proof: The recharging time on a charging link varies from 0 and $\bar{t}_{a}$. The latter does not depend on the link flow, and thus the maximum amount of energy possibly charged on the link is flowindependent. As previously mentioned, since the energy consumption is distance based, the usability of a path is flow-independent.

The above proposition essentially suggests that all the usable paths can be pre-specified for each O-D pair. We use $\hat{P}^{w}$ to denote the set of usable paths between O-D pair $w$, and further assume that at least one usable path exists for each O-D pair. Otherwise, there is no feasible flow distribution for the network of interest. 


\subsection{Choice of operating speed on charging link}

Consider an EV travels along path $p \in \hat{P}^{w}, w \in W$, and let $s_{a}^{p}$ denote the actual travel time that the vehicle spends on charging link $a \in \hat{A}$ along the path. The actual travel time is corresponding to the operating speed chosen by the vehicle. With Assumption v discussed in Section 2, we have $t_{a}\left(v_{a}\right) \leq s_{a}^{p} \leq \bar{t}_{a}$. Further, let $\hat{s}_{a}^{p}$ denote the corresponding recharging time and thus $\hat{s}_{a}^{p} \leq s_{a}^{p}$, because drivers do not necessarily charge when driving on a charging link.

As previously discussed, the EV driver attempts to complete his or her trip with the least time. By considering the SOC of the battery and the energy required for finishing the trip, the driver will decide a recharging plan that dictates his or her operating speed and recharging time. With a given traffic flow distribution $\widetilde{\boldsymbol{v}}$, the optimal decision of a driver traveling along path $p \in \hat{P}^{w}$ can be obtained by solving the following recharging time (RT) problem whose decision variables include recharging time, actual travel time and the SOC of the battery:

RT:

$$
\begin{array}{lc} 
& \min _{L, \hat{s}, s} \sum_{a \in \hat{A}(p)} s_{a}^{p}+\sum_{a \in A(p) \backslash \hat{A}(p)} t_{a}\left(\tilde{v}_{a}\right) \\
s_{a}^{p} \geq t_{a}\left(\tilde{v}_{a}\right) & \forall a \in \hat{A}(p) \\
s_{a}^{p} \leq \bar{t}_{a} & \forall a \in \hat{A}(p) \\
\hat{s}_{a}^{p} \leq s_{a}^{p} & \forall a \in \hat{A}(p) \\
L_{j}^{p}-L_{i}^{p}+d_{a} \varpi=0 & \forall(i, j)=a \in A(p) \backslash \hat{A}(p) \\
L_{j}^{p}-L_{i}^{p}+d_{a} \varpi-\hat{s}_{a}^{p} e=0 & \forall(i, j)=a \in \hat{A}(p) \\
L_{i}^{p} \leq L_{\max } & \forall i \in N(p) \\
L_{o(w)}^{p}=L_{0} & \\
\hat{s}_{a}^{p} \geq 0 & \forall a \in \hat{A}(p) \\
L_{i}^{p} \geq m^{w} & \forall i \in N(p)
\end{array}
$$

where $L_{\max }$ and $L_{0}$ represent the battery size and initial SOC; $\varpi$ and $e$ are respectively the energy consumption rate of the EV and recharging rate of charging lanes; $m^{w}$ represents a minimum SOC that the driver will feel comfortable with when operating the EV; such a reserved range represents the level of range anxiety of the driver; $L_{i}^{p}$ denotes the SOC at node $i \in N$ along path $p \in \hat{P}^{w} ; A(p)$ denotes the links along the path while $\hat{A}(p)$ denotes all charging links along the path; and $N(p)$ denotes the nodes of the path.

As formulated, the objective function is to minimize the total trip time along path $p$. Constraints (1), (2), and (3) specify the relationship among the prevailing travel time $t_{a}\left(\tilde{v}_{a}\right)$, maximum travel time $\bar{t}_{a}$, actual travel time $s_{a}^{p}$ and recharging time $\hat{s}_{a}^{p}$ on charging links. Note that, the maximum travel time $\bar{t}_{a}$ is considered from the safety aspect in our paper, and can be set to be sufficiently large, or just be omitted. Constraints (4) and (5) specify the relation between the battery SOC at the starting and ending nodes of any utilized link. Constraint (6) ensures that the SOC cannot exceed the battery size. Constraint (7) specifies the initial SOC. Constraint (8) 
ensures that the recharging time cannot be negative. Constraint (9) requires that the SOC cannot be lower than the reserved range.

$\mathrm{RT}$ is a linear program written for each usable path $p \in \hat{P}^{w}, w \in W$. Below are its optimality conditions:

(1)-(9)

\begin{tabular}{|c|c|}
\hline$-\bar{\kappa}_{i j}^{p}+\bar{\kappa}_{k i}^{p}+\bar{\xi}_{i}^{p}-\bar{\beta}_{i}^{p}=0$ & $\begin{array}{l}\forall(i, j),(k, i)=a \in A(p), \\
i \notin\{o(w), d(w)\}\end{array}$ \\
\hline$-\bar{\kappa}_{o(w) j}^{p}+\bar{\xi}_{o(w)}^{p}+\bar{\theta}_{o(w)}^{p}-\bar{\beta}_{o(w)}^{p}=0$ & $\forall(o(w), j)=a \in A(p)$ \\
\hline $\bar{\kappa}_{k d(w)}^{p}+\bar{\xi}_{d(w)}^{p}-\bar{\beta}_{d(w)}^{p}=0$ & $\forall(k, d(w))=a \in A(p)$ \\
\hline $\bar{\varphi}_{a}^{p}-e \bar{\kappa}_{a}^{p}-\bar{\alpha}_{a}^{p}=0$ & $\forall a \in \hat{A}(p)$ \\
\hline $1-\bar{\pi}_{a}^{p}+\bar{\eta}_{a}^{p}-\bar{\varphi}_{a}^{p}=0$ & $\forall a \in \hat{A}(p)$ \\
\hline$\left(s_{a}^{p}-t_{a}\left(v_{a}\right)\right) \bar{\pi}_{a}^{p}=0$ & $\forall a \in \hat{A}(p)$ \\
\hline$\left(s_{a}^{p}-\bar{t}_{a}\right) \bar{\eta}_{a}^{p}=0$ & $\forall a \in \hat{A}(p)$ \\
\hline$\left(\hat{s}_{a}^{p}-s_{a}^{p}\right) \bar{\varphi}_{a}^{p}=0$ & $\forall a \in \hat{A}(p)$ \\
\hline$\left(L_{i}^{p}-L_{\max }\right) \bar{\xi}_{i}^{p}=0$ & $\forall i \in N(p)$ \\
\hline$\hat{s}_{a}^{p} \bar{\alpha}_{a}^{p}=0$ & $\forall a \in \hat{A}(p)$ \\
\hline$\left(L_{i}^{p}-m^{w}\right) \bar{\beta}_{i}^{p}=0$ & $\forall i \in N(p)$ \\
\hline $\bar{\pi}_{a}^{p} \geq 0$ & $\forall a \in \hat{A}(p)$ \\
\hline $\bar{\eta}_{a}^{p} \geq 0$ & $\forall a \in \hat{A}(p)$ \\
\hline $\bar{\varphi}_{a}^{p} \geq 0$ & $\forall a \in \hat{A}(p)$ \\
\hline $\bar{\xi}_{i}^{p} \geq 0$ & $\forall i \in N(p)$ \\
\hline $\bar{\alpha}_{a}^{p} \geq 0$ & $\forall a \in \hat{A}(p)$ \\
\hline $\bar{\beta}_{i}^{p} \geq 0$ & $\forall i \in N(p)$ \\
\hline
\end{tabular}

where $\bar{\pi}_{a}^{p}, \bar{\eta}_{a}^{p}, \bar{\varphi}_{a}^{p}, \bar{\xi}_{i}^{p}, \bar{\theta}_{o(w)}^{p}, \bar{\alpha}_{a}^{p}$, and $\bar{\beta}_{i}^{p}$ are the multipliers associated with constraints (1), (2), (3), (6), (7), (8), and (9), while $\bar{\kappa}_{a}^{p}$ is the multiplier associated with (4) and (5).

\subsection{Definition and formulation of network equilibrium}

Definition 2 (He et al., 2014). At equilibrium, all utilized paths are usable. Travel times of utilized paths between an O-D pair are the same, but less than or equal to that of any unutilized usable path between the same O-D pair.

Mathematically, we can define the network equilibrium conditions as follows:

(1)-(26) 


$$
\begin{array}{ll}
\sum_{p \in \hat{P} w} f_{p}=g^{w} & \forall w \in W \\
v_{a}=\sum_{w \in W} \sum_{p \in \hat{P}^{w}} f_{p} \delta_{a}^{p} & \forall a \in A \\
f_{p} \geq 0 & \forall p \in \hat{P}^{w}, w \in W \\
\left(\sum_{a \in A(p) \backslash \hat{A}(p)} t_{a}\left(v_{a}\right)+\sum_{a \in \hat{A}(p)} s_{a}^{p}-\mu^{w}\right) f_{p}=0 & \forall p \in \hat{P}^{w}, w \in W \\
\sum_{a \in A(p) \backslash \hat{A}(p)} t_{a}\left(v_{a}\right)+\sum_{a \in \hat{A}(p)} s_{a}^{p} \geq \mu^{w} & \forall p \in \hat{P}^{w}, w \in W
\end{array}
$$

where $\mu^{w}$ is an auxiliary variable, representing the equilibrium trip time for O-D pair $w \in W$.

Some may argue that EV drivers will prefer a path containing charging links, since the presence of charging opportunities provide them a sense of security, mitigating or eliminating their range anxiety. To capture this effect, we can introduce a security factor $\zeta_{a} \geq 0$, which may increase with the distance of the charging link. Consequently, (30) and (31) can be rewritten as follows:

$$
\begin{array}{cc}
\left(\sum_{a \in A(p) \backslash \hat{A}(p)} t_{a}\left(v_{a}\right)+\sum_{a \in \hat{A}(p)} s_{a}^{p}-\sum_{a \in \hat{A}(p)} \zeta_{a}-\mu^{w}\right) f_{p}=0 & \forall p \in \hat{P}^{w}, w \in W \\
\sum_{a \in A(p) \backslash \hat{A}(p)} t_{a}\left(v_{a}\right)+\sum_{a \in \hat{A}(p)} s_{a}^{p}-\sum_{a \in \hat{A}(p)} \zeta_{a} \geq \mu^{w} & \forall p \in \hat{P}^{w}, w \in W
\end{array}
$$

To formulate an equivalent network equilibrium model, we first define a set $\Lambda=$ $\{(\boldsymbol{\pi}, \boldsymbol{\eta}, \boldsymbol{\varphi}, \boldsymbol{\kappa}, \boldsymbol{\xi}, \boldsymbol{\theta}, \boldsymbol{\zeta}, \boldsymbol{f}, \boldsymbol{v})\}$, where the vector satisfies the following conditions:

$$
\begin{array}{ll}
-\varphi_{a}^{p}-e \kappa_{a}^{p} \leq 0 & \forall p \in \hat{P}^{w}, w \in W, a \in \hat{A}(p) \\
-\kappa_{i j}^{p}+\kappa_{k i}^{p}-\xi_{i}^{p}+\varsigma_{i}^{p}=0 & \forall p \in \hat{P}^{w}, w \in W,(i, j),(k, i)=a \in A(p), \\
-\kappa_{o(w) j}^{p}-\xi_{o(w)}^{p}+\theta_{o(w)}^{p}+\varsigma_{o(w)}^{p}=0 & \forall \notin\{o(w), d(w)\} \\
\kappa_{k d(w)}^{p}-\xi_{d(w)}^{p}+\varsigma_{d(w)}^{p}=0 & \forall p \in \hat{P}^{w}, w \in W,(o(w), j)=a \in A(p) \\
\pi_{a}^{p}-\eta_{a}^{p}+\varphi_{a}^{p}=1 & \forall p \in \hat{P}^{w}, w \in W,(k, d(w))=a \in A(p) \\
\pi_{a}^{p} \geq 0 & \forall p \in \hat{P}^{w}, w \in W, a \in \hat{A}(p) \\
\eta_{a}^{p} \geq 0 & \forall p \in \hat{P}^{w}, w \in W, a \in \hat{A}(p) \\
& \forall p \in \hat{P}^{w}, w \in W, a \in \hat{A}(p)
\end{array}
$$


$\varphi_{a}^{p} \geq 0$

$$
\begin{aligned}
& \forall p \in \hat{P}^{w}, w \in W, a \in \hat{A}(p) \\
& \forall p \in \hat{P}^{w}, w \in W, i \in N(p)
\end{aligned}
$$$$
\varsigma_{i}^{p} \geq 0
$$

Proposition 2. The network equilibrium conditions (1)-(31) are equivalent to finding $\left(\boldsymbol{\pi}^{*}, \boldsymbol{\eta}^{*}, \boldsymbol{\varphi}^{*}, \boldsymbol{\kappa}^{*}, \boldsymbol{\xi}^{*}, \boldsymbol{\theta}^{*}, \boldsymbol{\zeta}^{*}, \boldsymbol{f}^{*}, \boldsymbol{v}^{*}\right) \in \Lambda$ that solves the following variational inequality:

NE-VI:

$$
\begin{aligned}
\sum_{p \in \hat{P}^{w}, w \in W}( & \sum_{a \in A(p) \backslash \hat{A}(p)} t_{a}^{*}+\sum_{a \in \hat{A}(p)} t_{a}^{*} \pi_{a}^{p *}-\sum_{a \in \hat{A}(p)} \bar{t}_{a} \eta_{a}^{p *}-\sum_{a \in A(p)} d_{a} \varpi \kappa_{a}^{p *}-\sum_{i \in N(p)} L_{m a x} \xi_{i}^{p *} \\
& \left.+L_{o} \theta_{o(w)}^{p *}+\sum_{i \in N(p)} m^{w} \varsigma_{i}^{p *}\right)\left(f_{p}-f_{p}^{*}\right) \\
& +\sum_{p \in \hat{P} \hat{P}^{w}, w \in W}\left[-\sum_{a \in \hat{A}(p)} t_{a}^{*}\left(\pi_{a}^{p}-\pi_{a}^{p *}\right)+\sum_{a \in \hat{A}(p)} \bar{t}_{a}\left(\eta_{a}^{p}-\eta_{a}^{p *}\right)\right. \\
& +\sum_{a \in A(p)} d_{a} \varpi\left(\kappa_{a}^{p}-\kappa_{a}^{p *}\right)+\sum_{i \in N(p)} L_{\max }\left(\xi_{i}^{p}-\xi_{i}^{p *}\right)-L_{o}\left(\theta_{o(w)}^{p}-\theta_{o(w)}^{p *}\right) \\
& \left.-\sum_{i \in N(p)} m^{w}\left(\varsigma_{i}^{p}-\varsigma_{i}^{p *}\right)\right] \geq 0, \forall(\boldsymbol{\pi}, \boldsymbol{\eta}, \boldsymbol{\varphi}, \boldsymbol{\kappa}, \boldsymbol{\xi}, \boldsymbol{\theta}, \boldsymbol{\varsigma}, \boldsymbol{f}, \boldsymbol{v}) \in \Lambda
\end{aligned}
$$

The equivalence can be established by expressing the optimality conditions of NE-VI and comparing them with the network equilibrium conditions. See Appendix B for the proof.

Proposition 3. NE-VI has at least one solution.

Proof: We construct a restricted NE-VI problem with a compact and convex feasible region. Given that all the functions are continuous, the restricted NE-VI admits at least one solution (see, e.g., Harker and Pang, 1990). Consequently, the original NE-VI has at least one solution.

Note that $\bar{\pi}_{a}^{p}, \bar{\eta}_{a}^{p}, \bar{\varphi}_{a}^{p}, \bar{\xi}_{i}^{p}, \bar{\theta}_{o(w)}^{p}, \bar{\beta}_{i}^{p}$, and $\bar{\kappa}_{a}^{p}$ are multipliers of RT, a linear problem written for each usable path with a compact and convex feasible region. Since the optimal value of RT is finite and bounded, we can always find some upper and lower bounds for these multipliers, within which optimal values of these multipliers still exist. From Appendix B, we have $\pi_{a}^{p}=\bar{\pi}_{a}^{p}, \eta_{a}^{p}=\bar{\eta}_{a}^{p}, \varphi_{a}^{p}=\bar{\varphi}_{a}^{p}, \kappa_{i j}^{p}=-\bar{\kappa}_{i j}^{p}, \xi_{i}^{p}=\bar{\xi}_{i}^{p}, \theta_{o(w)}^{p}=-\bar{\theta}_{o(w)}^{p}$, and $\varsigma_{i}^{p}=\bar{\beta}_{i}^{p}$. We can thus construct a restricted NE-VI by adding corresponding upper and lower bounds to $(\boldsymbol{\pi}, \boldsymbol{\eta}, \boldsymbol{\varphi}, \boldsymbol{\kappa}, \boldsymbol{\xi}, \boldsymbol{\theta}, \boldsymbol{\zeta})$. In addition, path and link flows $\boldsymbol{f}$ and $\boldsymbol{v}$ are bounded. Therefore, the restricted NE-VI problem has a compact and convex feasible region, and its optimal solutions also solve the original NE-VI problem. 
Different from traditional user equilibrium model, even if link performance functions are strictly monotone, the link flow solution from NE-VI may not be unique. To see this, consider the network in Fig. 2 with two parallel charging links and an O-D demand of 5. The travel time functions of the two links are $t_{1}\left(v_{1}\right)=6.4+0.1 v_{1}$, and $t_{2}\left(v_{2}\right)=6.3+0.1 v_{2} \mathrm{~min}$, and their distances are both $7.5 \mathrm{mi}$. Suppose that the battery size of an EV is $24 \mathrm{kWh}$, and its initial SOC is $0.15 \mathrm{kWh}$. The energy consumption rate is $0.29 \mathrm{kWh} / \mathrm{mi}$ and the recharging rate is 0.3 $\mathrm{kWh} / \mathrm{min}$. It is easy to verify that $v_{1}=3.5, v_{2}=1.5$; and $v_{1}=0.5, v_{2}=4.5$ are both user equilibrium link flows. In both cases, the travel times are $6.75 \mathrm{~min}$, equal to the recharging times. Note that any convex combination of these two solutions is also a user equilibrium link flow solution. However, all these solutions yield the same O-D equilibrium travel time.

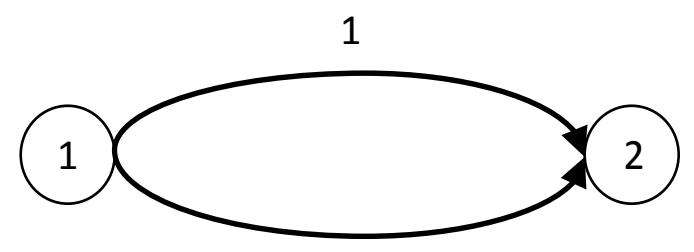

2

Fig. 2. A toy network with two nodes

\subsection{Solution procedure}

In this section, we solve NE-VI by reformulating it to be the following nonlinear optimization problem via a technique proposed by Aghassi et al. (2006):

NE-NLP:

$$
\begin{aligned}
& \min _{\boldsymbol{\pi}, \boldsymbol{\eta}, \boldsymbol{\varphi}, \boldsymbol{\kappa}, \xi, \boldsymbol{\theta}, \boldsymbol{\zeta}, \boldsymbol{f}, \boldsymbol{v}, \widetilde{\boldsymbol{g}}, \tilde{\boldsymbol{\pi}}, \tilde{\boldsymbol{\varphi}}, \tilde{\xi}} \sum_{p \in \hat{P}^{w}, w \in W}\left(\sum_{a \in A(p) \backslash \hat{A}(p)} t_{a}+\sum_{a \in \hat{A}(p)} t_{a} \pi_{a}^{p}-\sum_{a \in \hat{A}(p)} \bar{t}_{a} \eta_{a}^{p}-\sum_{a \in A(p)} d_{a} \varpi \kappa_{a}^{p}\right. \\
& \left.-\sum_{i \in N(p)} L_{\max } \xi_{i}^{p}+L_{o} \theta_{o(w)}^{p}+\sum_{i \in N(p)} m^{w} \varsigma_{i}^{p}\right) f_{p} \\
& -\sum_{p \in \hat{P}^{w}, w \in W}\left[\sum_{a \in \hat{A}(p)} t_{a} \pi_{a}^{p}+\sum_{a \in \hat{A}(p)} \bar{t}_{a} \eta_{a}^{p}-\sum_{a \in A(p)} d_{a} \varpi \kappa_{a}^{p}+\sum_{i \in N(p)} L_{\max } \xi_{i}^{p}\right. \\
& \left.+L_{o} \theta_{o(w)}^{p}+\sum_{i \in N(p)} m^{w} S_{i}^{p}\right]-\sum_{w \in W} g^{w} \tilde{g}^{w}-\sum_{p \in \hat{P}^{w}, w \in W} \sum_{a \in \hat{A}(p)} \tilde{\pi}_{a}^{p}
\end{aligned}
$$

s.t. (27)-(29), (32)-(41)
$\tilde{\pi}_{a}^{p} \leq-t_{a}$
$\forall p \in \hat{P}^{w}, w \in W, a \in \hat{A}(p)$
$-\tilde{\pi}_{a}^{p} \leq \bar{t}_{a}$
$\forall p \in \hat{P}^{w}, w \in W, a \in \hat{A}(p)$ 


$$
\begin{array}{lr}
\tilde{\varphi}_{a}^{p}+\tilde{\pi}_{a}^{p} \leq 0 & \forall p \in \hat{P}^{w}, w \in W, a \in \hat{A}(p) \\
-\tilde{\xi}_{i}^{p}+\tilde{\xi}_{j}^{p}=d_{a} \varpi & \forall p \in \hat{P}^{w}, w \in W,(i, j)=a \in A \backslash \hat{A}(p) \\
-\tilde{\xi}_{i}^{p}+\tilde{\xi}_{j}^{p}+e \tilde{\varphi}_{a}^{p}=d_{a} \varpi & \forall p \in \hat{P}^{w}, w \in W,(i, j)=a \in \hat{A}(p) \\
-\tilde{\xi}_{i}^{p} \leq L_{\max } & \forall p \in \hat{P}^{w}, w \in W, i \in N(p) \\
\tilde{\xi}_{o(w)}^{p}=-L_{o} & \forall p \in \hat{P}^{w}, w \in W \\
\tilde{\xi}_{i}^{p} \leq-m^{w} & \forall p \in \hat{P}^{w}, w \in W, i \in N(p) \\
\sum_{a \in A(p) \backslash \hat{A}(p)} t_{a}+\sum_{a \in \hat{A}(p)} t_{a} \pi_{a}^{p}-\sum_{a \in \hat{A}(p)} \bar{t}_{a} \eta_{a}^{p} & \\
-\sum_{a \in A(p)} d_{a} \varpi \kappa_{a}^{p}-\sum_{i \in N(p)} L_{m a x} \xi_{i}^{p} & \forall p \in \hat{P}^{w}, w \in W \\
+L_{o(w)} \theta_{o(w)}^{p}+\sum_{i \in N(p)} m^{w} s_{i}^{p} \geq \tilde{g}^{w} &
\end{array}
$$

$\tilde{\varphi}_{a}^{p} \geq 0$

$\forall p \in \hat{P}^{w}, w \in W, a \in \hat{A}(p)$

where $\tilde{\varphi}_{a}^{p}, \tilde{\xi}_{i}^{p}, \tilde{\pi}_{a}^{p}$, and $\tilde{g}^{w}$ are auxiliary variables.

In the above, the objective function is to minimize the gap between a primal and dual problem associated with NE-VI, and the constraints are those from the primal and dual problems. The optimal solution $\left(\boldsymbol{\pi}^{*}, \boldsymbol{\eta}^{*}, \boldsymbol{\varphi}^{*}, \boldsymbol{\kappa}^{*}, \boldsymbol{\xi}^{*}, \boldsymbol{\theta}^{*}, \boldsymbol{\varphi}^{*}, \boldsymbol{f}^{*}, \boldsymbol{v}^{*}\right)$ to NE-NLP solves NE-VI if the gap becomes zero. Given all the usable paths, NE-NLP is a regular nonlinear optimization problem and can be easily solved by commercial nonlinear solvers such as CONOPT (Drud, 1994). However, since it is time consuming to enumerate all usable paths, we apply a columngeneration procedure developed by He et al. (2014). The procedure starts with a subset of $\hat{P}^{w}$, $w \in W$, and solves a restricted version of NE-NLP defined upon the subset. Another subproblem is then solved to determine whether the solution to the restricted NE-NLP solves the original formulation. If not, a new usable path generated from solving the sub-problem will be added to the subset of $\hat{P}^{w}$ and the iteration proceeds until termination.

Some new variables are introduced to formulate the sub-problem. Specifically, let $F_{a}^{w}$ denote the amount of electricity recharged on charging link $a$ to an EV traveling between O-D pair $w \in W$; let $\Delta$ be the node-link incidence matrix associated with the network and $E^{w}$ is a vector with a length of $|N|$. The vector consists of two-nonzero components: one has a value of 1 in the component corresponding to the origin of $w$ and the other has a value of -1 in the component corresponding to the destination of $w$. Given the link flow solution $\widetilde{\boldsymbol{v}}$ obtained from solving the restricted NE-NLP, the sub-problem of finding shortest usable path can be formulated as follows for each O-D pair $w \in W$ :

SP:

$$
\min _{\widehat{\boldsymbol{s}}, \boldsymbol{x}, \boldsymbol{s}} \sum_{a \in \hat{A}} s_{a}^{w} x_{a}^{w}+\sum_{a \in A \backslash \hat{A}} t_{a}\left(\tilde{v}_{a}\right) x_{a}^{w}
$$




$$
\begin{array}{ll}
L_{j}^{w}-L_{i}^{w}+d_{a} \varpi-F_{a}^{w}=\psi_{a}^{w} & \forall(i, j)=a \in A \\
-K\left(1-x_{a}^{w}\right) \leq \psi_{a}^{w} \leq K\left(1-x_{a}^{w}\right) & \forall a \in A \\
m^{w} \leq L_{i}^{w} \leq L_{\text {max }} & \forall i \in N \\
F_{a}^{w}=0 & \forall a \in A \backslash \hat{A} \\
F_{a}^{w}=\hat{s}_{a}^{w} e & \forall a \in \hat{A} \\
s_{a}^{w} \geq t_{a}\left(\tilde{v}_{a}\right) & \forall a \in \hat{A} \\
s_{a}^{w} \leq \bar{t}_{a} & \forall a \in \hat{A} \\
\hat{s}_{a}^{w} \leq & \forall a \in \hat{A} \\
\hat{s}_{a}^{w} \geq 0 & \forall a \in \hat{A} \\
L_{o(w)}^{w}=L_{0} & \\
x_{a}^{w} \in\{0,1\} & \forall a \in A
\end{array}
$$

where $x_{a}^{w}$ is a binary variable, which is equal to 1 if link $a$ is utilized and 0 otherwise; $\psi_{a}^{w}$ is a variable, which equals 0 if link $a$ is utilized and is unrestricted otherwise; $K$ is a sufficiently large constant; $s_{a}^{w}$ and $\hat{s}_{a}^{w}$ are the actual travel time and recharging time on charging link $a$ of OD pair $w$.

In the above, the objective function is to minimize the total trip time. Constraint (42) ensures flow balance. Constraints (43) and (44) specify the relation between the battery SOC at the starting and ending nodes of any utilized link. Constraint (45) ensures that the SOC can be neither lower than the reserved range nor higher than the battery size. Constraints (46) and (47) suggest that EVs can only recharge at charging links, and the amount of energy recharged is equal to the recharging rate multiplying the charging time. Constraints (48)-(50) specify the relationship among the prevailing travel time, actual travel time, recharging time and maximum travel time on charging links. Constraint (51) ensures that recharging time must be nonnegative. Constraint (52) specifies the initial SOC. Finally, constraint (53) ensures that $x_{a}^{w}$ is binary.

As formulated, SP is a mixed integer nonlinear problem, not easy to solve, particularly for a large network. We linearize it to be the following equivalent formulation by introducing a new variable $z_{a}^{w}$ :

L-SP:

$$
\min _{\hat{\boldsymbol{s}}, \boldsymbol{x}, \boldsymbol{s}, \boldsymbol{Z}, \boldsymbol{L}, \boldsymbol{F}, \boldsymbol{\rho}} \sum_{a \in \hat{A}} z_{a}^{w}+\sum_{a \in A \backslash \hat{A}} t_{a}\left(\tilde{v}_{a}\right) x_{a}^{w}
$$

s.t. (42)-(53)

$$
\begin{array}{lr}
z_{a}^{w} \leq \bar{t}_{a} x_{a}^{w} & \forall a \in \hat{A} \\
z_{a}^{w} \geq 0 & \forall a \in \hat{A} \\
z_{a}^{w} \leq s_{a}^{w} & \forall a \in \hat{A} \\
z_{a}^{w} \geq \bar{t}_{a} x_{a}^{w}+s_{a}^{w}-\bar{t}_{a} & \forall a \in \hat{A}
\end{array}
$$

L-SP is a mixed integer linear program that can be easily solved using commercial solvers such as CPLEX 12.2. For each O-D pair $w \in W$, the optimal solution to L-SP, denoted as 
$\widetilde{\boldsymbol{x}}^{w}$, can be used to construct a shortest usable path, i.e., $\tilde{p}^{w}$. The iterative solution procedure for solving NE-VI can thus be written as follows:

Step 0: For each O-D pair $w \in W$, solve L-SP with $\widetilde{\boldsymbol{v}}=\mathbf{0}$. Construct $\widetilde{P}^{w}=\left\{\tilde{p}^{w}\right\}$.

Step 1: Solve NE-NLP upon $\widetilde{P}^{w}$. Denote $\widetilde{\boldsymbol{v}}$ as the optimal solution, and $\mu^{w}$ as multipliers associated with constraints $\sum_{p \in \tilde{P}^{w}} f_{p}=g^{w}, \forall w \in W$.

Step 2: For each O-D pair $w \in W$, solve L-SP and obtain a path $\tilde{p}^{w}$. For $w \in W$, if $\sum_{a \in \hat{A}\left(\tilde{p}^{w}\right)} z_{a}^{w}+\sum_{a \in A\left(\tilde{p}^{w}\right) \backslash \hat{A}\left(\tilde{p}^{w}\right)} t_{a}\left(\tilde{v}_{a}\right) x_{a}^{w}<\mu^{w}$, add $\tilde{p}^{w}$ to $\tilde{P}^{w}$. If $\sum_{a \in \hat{A}\left(\tilde{p}^{w}\right)} z_{a}^{w}+\sum_{a \in A\left(\tilde{p}^{w}\right) \backslash \hat{A}\left(\tilde{p}^{w}\right)} t_{a}\left(\tilde{v}_{a}\right) x_{a}^{w} \geq \mu^{w}$ for all O-D pairs, stop and $\widetilde{v}$ is the equilibrium path flow distribution. Otherwise, go to Step 1.

\subsection{Numerical examples}

Numerical examples in this section are based on the Nguyent-Dupius network (see Fig. 3), which consists of 13 nodes, 19 links, and 4 O-D pairs. The link performance function is assumed to be $t_{a}\left(v_{a}\right)=a_{0}+b_{0} \times v_{a}$ min where $a_{0}$ and $b_{0}$ are presented in Table 1 together with other link characteristics. The minimum speed limit is $30 \mathrm{mph}$ and the O-D demands can be found in Nguyen and Dupius (1984).

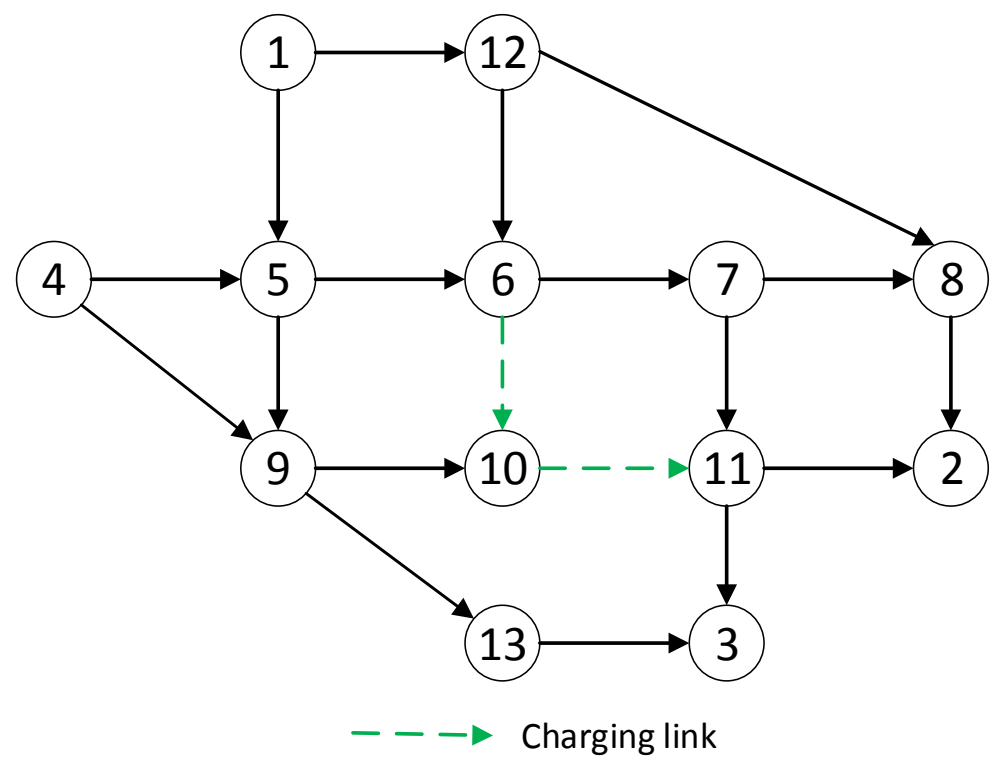

Fig. 3 Nguyen-Dupius network 
Table 1 Nguyen-Dupius network characteristics

\begin{tabular}{cccccccc}
\hline Link & $a_{0}(\mathrm{~min})$ & $b_{0}$ & Length $(\mathrm{mi})$ & Link & $a_{0}(\mathrm{~min})$ & $b_{0}$ & Length $(\mathrm{mi})$ \\
\hline $1-5$ & 11.06 & 0.0075 & 14.70 & $8-2$ & 14.22 & 0.0125 & 18.90 \\
$1-12$ & 14.22 & 0.01 & 18.90 & $9-10$ & 15.8 & 0.005 & 21.00 \\
$4-5$ & 14.22 & 0.01 & 18.90 & $9-13$ & 14.22 & 0.005 & 18.90 \\
$4-9$ & 18.96 & 0.005 & 25.20 & $10-11$ & 9.48 & 0.0025 & 12.60 \\
$5-6$ & 4.74 & 0.0025 & 6.30 & $11-2$ & 14.22 & 0.005 & 18.90 \\
$5-9$ & 14.22 & 0.0075 & 18.90 & $11-3$ & 12.64 & 0.0075 & 16.80 \\
$6-7$ & 7.90 & 0.01 & 10.50 & $12-6$ & 11.06 & 0.0025 & 14.70 \\
$6-10$ & 20.54 & 0.005 & 27.30 & $12-8$ & 22.12 & 0.01 & 29.40 \\
$7-8$ & 7.90 & 0.0125 & 10.50 & $13-3$ & 17.38 & 0.01 & 23.10 \\
$7-11$ & 14.22 & 0.0125 & 18.90 & & & & \\
\hline
\end{tabular}

As per the specification of Nissan Leaf 2013, $L_{\text {max }}$ is set to be $24 \mathrm{kWh}$ and the energy consumption rate $\varpi$ is $0.29 \mathrm{kWh} / \mathrm{mi}$ (Nissan USA, 2013; US Department of Energy, 2013). The reserved range, i.e., $m^{w}$, is 0 and the initial SOC, i.e., $L_{0}$, is $20 \mathrm{kWh}$. We first consider a scenario with a low recharging rate $e=0.1 \mathrm{kWh} / \mathrm{min}$, a level similar to a level 2 charger (see Table 2). We obtain an equilibrium solution shown in Table 3 and Table 4. From Table 4, we can observe that the energy consumptions along all utilized paths without recharging are less than or equal to the initial SOC, i.e., $20 \mathrm{kWh}$; while along the paths with recharging (e.g., path 3 and 5), they are exactly equal to the sum of the initial SOC and the energy recharged. Fig. 4 shows the battery SOC, cumulative energy recharged, and consumption at the nodes along path 3 . In this particular solution, drivers of path 3 will only recharge the amount of energy just sufficient to support their trips.

Fig. 5 illustrates the prevailing, actual travel times and recharging time on two charging links along path 3 and 5. It can be observed that, when the recharging time is no greater than the prevailing travel time (see link 6-10 and 10-11 in path 3, and link 6-10 in path 5), the actual travel time is equal to the prevailing travel time. This implies that given the recharging time is sufficient, drivers will travel at the prevailing speed. On the other hand, if the recharging time is greater than the prevailing travel time (see link 10-11 in path 5), the actual travel time is equal to the recharging time, as drivers have to slow down to ensure sufficient recharging time.

Table 2 BEV charger specification (He et al., 2014)

\begin{tabular}{cccc}
\hline Charging level & Level 1 & Level 2 & Level 3 \\
\hline Power $(\mathrm{kW})$ & 1.44 & 6 & 90 \\
Charging circuit & $120 \mathrm{~V}, 15 \mathrm{~A}$ & $240 \mathrm{~V}, 30 \mathrm{~A}$ & $500 \mathrm{~V}, 200 \mathrm{~A}$ \\
\hline
\end{tabular}


Table 3 Equilibrium link flow (veh/h)

\begin{tabular}{cccccc}
\hline Link & Link flow & Link & Link flow & Link & Link flow \\
\hline $1-5$ & 800.00 & $6-1$ & 756.16 & $11-2$ & 294.78 \\
$1-12$ & 400.00 & $7-8$ & 305.22 & $11-3$ & 800.00 \\
$4-5$ & 600.00 & $7-11$ & 338.62 & $12-6$ & 0 \\
$4-9$ & 200.00 & $8-2$ & 705.22 & $12-8$ & 400.00 \\
$5-6$ & 1400.00 & $9-10$ & 0 & $13-3$ & 200.00 \\
$5-9$ & 0 & $9-13$ & 200.00 & & \\
$6-7$ & 643.84 & $10-11$ & 756.16 & & \\
\hline
\end{tabular}

Table 4 Path-related information of the equilibrium flow pattern

\begin{tabular}{cclcccccc}
\hline OD & $\begin{array}{c}\text { Path } \\
\text { ID }\end{array}$ & Node sequence & $\begin{array}{c}\text { Path } \\
\text { flow } \\
\text { (veh) }\end{array}$ & $\begin{array}{c}\text { Path } \\
\text { travel } \\
\text { time } \\
(\mathrm{min})\end{array}$ & $\begin{array}{c}\text { Energy } \\
\text { consumption } \\
(\mathrm{kWh})\end{array}$ & $\begin{array}{c}\text { Link 6-10 } \\
\text { recharging } \\
\text { time (min) }\end{array}$ & $\begin{array}{c}\text { Link 10-11 } \\
\text { recharging } \\
\text { time (min) }\end{array}$ & $\begin{array}{c}\text { Energy } \\
\text { recharged } \\
(\mathrm{kWh})\end{array}$ \\
\hline $1-2$ & 1 & $1-12-8-2$ & 400.00 & 77.13 & 19.49 & 0 & 0 & 0 \\
$1-3$ & 2 & $1-5-6-7-11-3$ & 338.62 & 91.91 & 19.49 & 0 & 0 & 0 \\
& 3 & $1-5-6-10-11-3$ & 461.38 & 91.91 & 22.53 & 12.86 & 12.47 & 2.53 \\
$4-2$ & 4 & $4-5-6-7-8-2$ & 305.22 & 94.12 & 18.88 & 0 & 0 & 0 \\
& 5 & $4-5-6-10-11-2$ & 294.78 & 94.12 & 24.36 & 26.51 & 17.09 & 4.36 \\
$4-3$ & 6 & $4-9-13-3$ & 200.00 & 56.88 & 19.49 & 0 & 0 & 0 \\
\hline
\end{tabular}

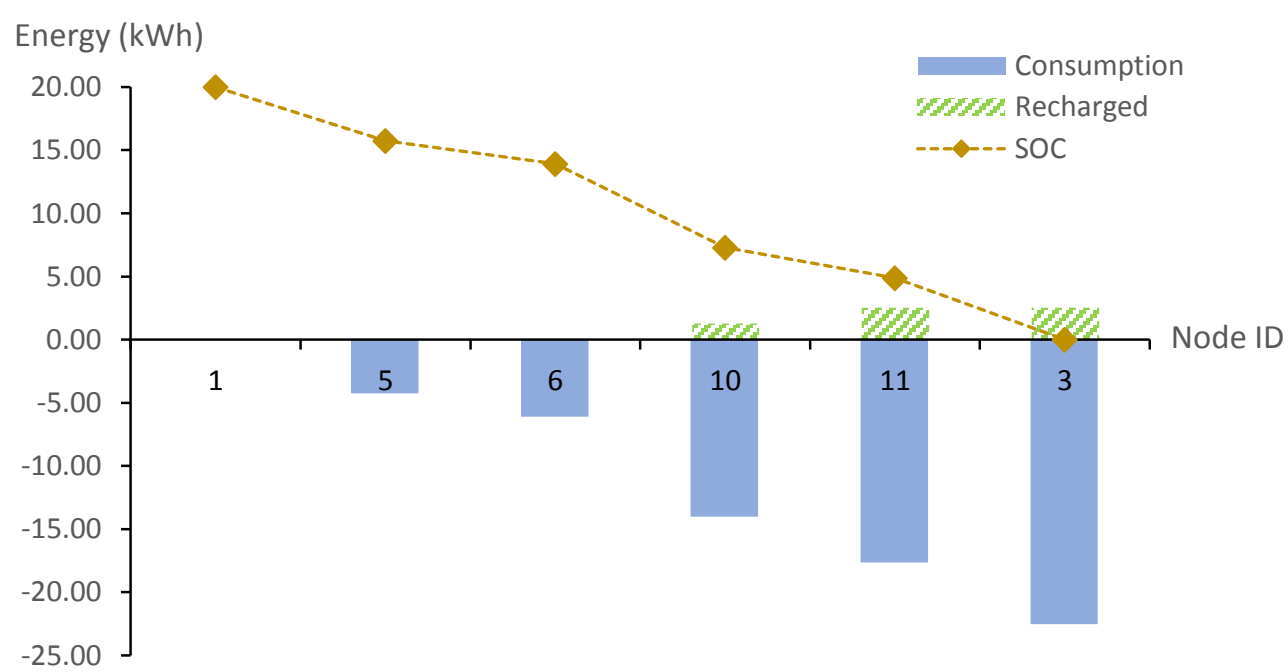

Fig. 4 SOC, cumulative energy recharged, and consumption at nodes along path 3 

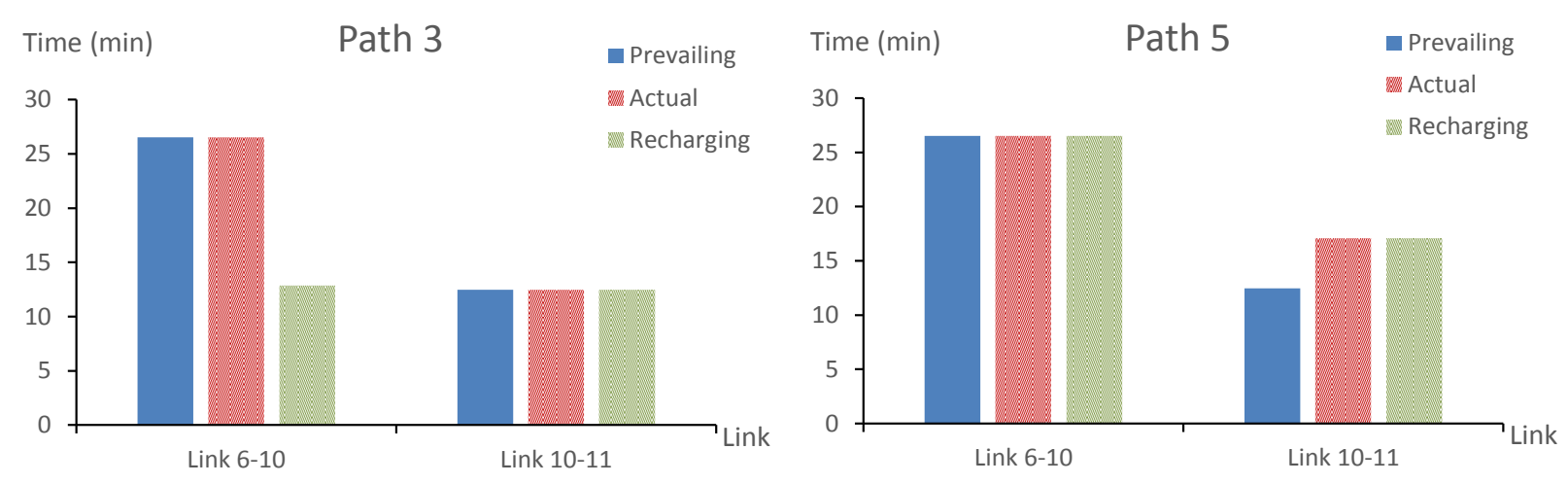

Fig. 5 Prevailing, actual travel time and recharging time

To analyze the impacts of the energy recharging rate, we further consider another scenario with $e=1.5 \mathrm{kWh} / \mathrm{min}$, a rate comparable to a level 3 charger (see Table 2). Similarly, the equilibrium flow pattern is obtained as shown in Table 5. Comparing Table 4 and Table 5, we can observe that path 5 has changed, since path 4-5-6-10-11-2 is no longer one of the shortest paths between O-D pair 4-2. Further, although path 3 remains the same and the recharged energy is also the same, the recharging time and the charging links EVs choose to recharge on are different. Specifically, the recharging time is only $1.69 \mathrm{~min}$, much less than the time of 25.33 min in Table 4; EVs are only charged on link 10-11 (see Table 5 and Fig. 6). As expected, a higher recharging rate yields less recharging time and different choice of charging links. When recharging becomes more efficient, drivers may not need to slow down to recharge, thereby reducing actual travel time and thus the total system travel time. Indeed, the total travel time reduces from 172,227 to $156,994 \mathrm{~min}$, while the recharging time reduces from 24,539 to 1,540 min in the scenarios considered above.

Table 5 Path-related information of the equilibrium flow pattern

\begin{tabular}{cclcccccc}
\hline OD & $\begin{array}{c}\text { Path } \\
\text { ID }\end{array}$ & $\begin{array}{c}\text { Node } \\
\text { sequence }\end{array}$ & $\begin{array}{c}\text { Path } \\
\text { flow } \\
\text { (veh) }\end{array}$ & $\begin{array}{c}\text { Path } \\
\text { travel } \\
\text { time } \\
(\mathrm{min})\end{array}$ & $\begin{array}{c}\text { Energy } \\
\text { consumption } \\
(\mathrm{kWh})\end{array}$ & $\begin{array}{c}\text { Link 6-10 } \\
\text { recharging } \\
\text { time (min) }\end{array}$ & $\begin{array}{c}\text { Link 10-11 } \\
\text { recharging } \\
\text { time (min) }\end{array}$ & $\begin{array}{c}\text { Energy } \\
\text { recharging } \\
(\mathrm{kWh})\end{array}$ \\
\hline $1-2$ & 1 & $1-12-8-2$ & 400.00 & 73.51 & 19.49 & 0 & 0 & 0 \\
$1-3$ & 2 & $1-5-6-7-11-3$ & 365.85 & 88.10 & 19.49 & 0 & 0 & 0 \\
& 3 & $1-5-6-10-11-3$ & 434.15 & 88.10 & 22.53 & 0 & 1.69 & 2.53 \\
$4-2$ & 4 & $4-5-6-7-8-2$ & 121.95 & 74.97 & 18.88 & 0 & 0 & 0 \\
& 5 & $4-9-10-11-2$ & 478.05 & 74.97 & 22.53 & 0 & 1.69 & 2.53 \\
$4-3$ & 6 & $4-9-13-3$ & 200.00 & 60.66 & 19.49 & 0 & 0 & 0 \\
\hline
\end{tabular}




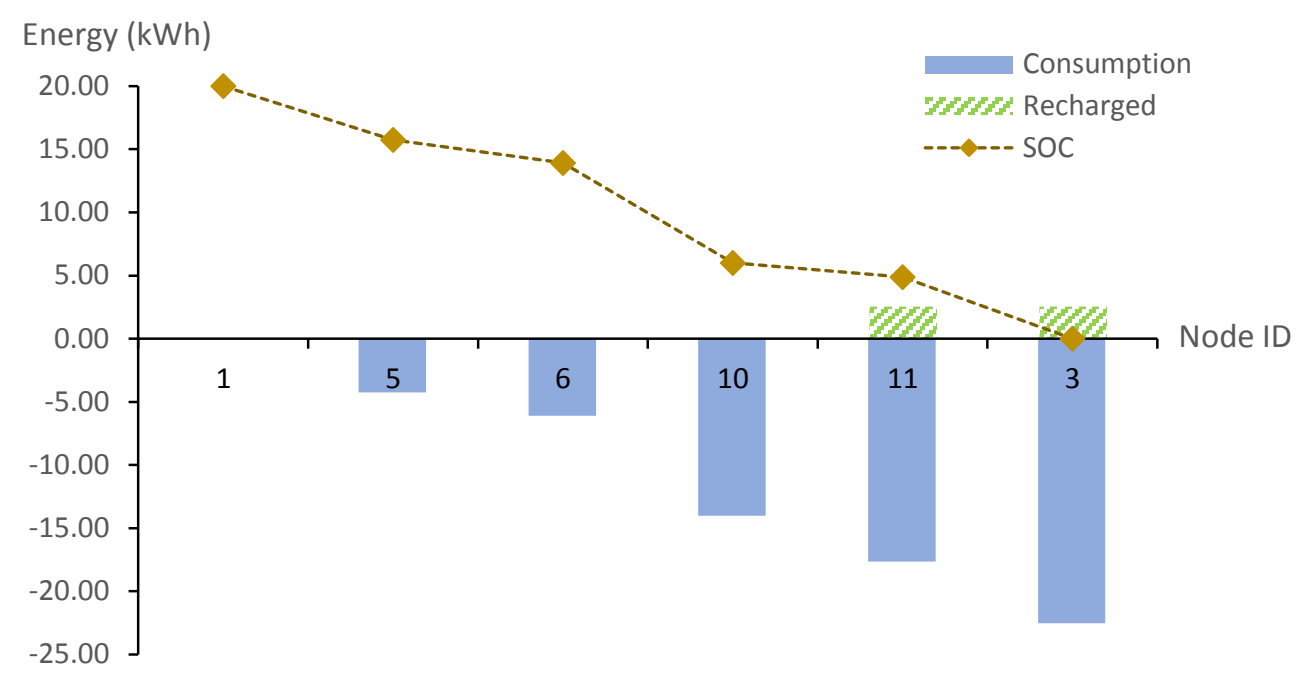

Fig. 6 SOC, cumulative energy recharged, and consumption at nodes along path 3

To analyze the impact of the initial SOC of battery and the reserved range on the equilibrium, we solve NE-NLP with $L_{0}=18,20$, and $22 \mathrm{kWh}$ and $m^{w}=0,1,2$, and $3 \mathrm{kWh}$. Their social costs, i.e., the total system travel times, and recharging times are presented in Table 6. All results are within expectation, demonstrating that the proposed model can reasonably capture the impacts of various parameters.

Table 6 Social cost and recharging time

\begin{tabular}{lcc}
\hline & Social cost (min) & Recharging time (min) \\
\hline$m^{w}=0 \mathrm{kWh}$ and $e=1.5 \mathrm{kWh} / \mathrm{min}$ & & \\
\hline$L_{0}=18 \mathrm{kWh} / \mathrm{min}$ & 178,066 & 5,404 \\
$L_{0}=20 \mathrm{kWh} / \mathrm{min}$ & 156,994 & 1,540 \\
$L_{0}=22 \mathrm{kWh} / \mathrm{min}$ & 152,159 & 246 \\
\hline$L_{0}=22 \mathrm{kWh} / \mathrm{min}$ and $e=1.5 \mathrm{kWh} / \mathrm{min}$ & & \\
\hline$m^{w}=0 \mathrm{kWh}$ & 152,159 & 246 \\
$m^{w}=1 \mathrm{kWh}$ & 156,994 & 932 \\
$m^{w}=2 \mathrm{kWh}$ & 156,994 & 1,540 \\
$m^{w}=3 \mathrm{kWh}$ & 178,142 & 4,187 \\
\hline
\end{tabular}

\section{Locating Charging Lanes}

\subsection{Model formulation}

Given a limited budget, we now optimize the locations of charging lanes in a general road network to minimize the social cost. Denote $y_{a}$ as a binary variable, representing whether link $a$ should be converted into a charging link. If yes, $y_{a}=1$; otherwise, $y_{a}=0$. The charging-lane 
location problem (CLL) can be formulated as follows. For completeness, we repeat some previously presented constraints here.

\section{CLL:}

$$
\min _{\boldsymbol{\pi}, \boldsymbol{\eta}, \boldsymbol{\varphi}, \mathbf{k}, \boldsymbol{\xi}, \boldsymbol{\theta}, \boldsymbol{\zeta}, \boldsymbol{\beta}, \boldsymbol{\gamma}, \boldsymbol{\lambda}, \boldsymbol{f}, \boldsymbol{v}, \boldsymbol{\rho}, \boldsymbol{y}} \sum_{w \in W} \rho^{w} g^{w}
$$

s.t. (27)-(29)

$$
\sum_{a \in A} c_{a} d_{a} y_{a} \leq I
$$

$y_{a} \in\{0,1\}$

$\forall a$

$\sum_{a \in A(p)} t_{a}\left(1-y_{a}\right)+\sum_{a \in A(p)} \gamma_{a}^{p} y_{a}-\rho^{w} \geq 0$

$\forall p \in \hat{P}^{w}, w \in W$

$y_{a}\left(-\varphi_{a}^{p}-e \kappa_{a}^{p}\right) \leq 0$

$\forall p \in \hat{P}^{w}, w \in W, a \in A(p)$

$-\kappa_{i j}^{p}+\kappa_{k i}^{p}-\xi_{i}^{p}+\varsigma_{i}^{p}=0$

$\forall p \in \hat{P}^{w}, w \in W,(i, j),(k, i)=a$

$\in A(p), i \notin\{o(w), d(w)\}$

$-\kappa_{o(w) j}^{p}-\xi_{o(w)}^{p}+\theta_{o(w)}^{p}+\varsigma_{o(w)}^{p}=0$

$\forall p \in \hat{P}^{w}, w \in W,(o(w), j)=a$

$\in A(p)$

$\kappa_{k d(w)}^{p}-\xi_{d(w)}^{p}+\varsigma_{d(w)}^{p}=0$

$\forall p \in \hat{P}^{w}, w \in W,(k, d(w))=a$

$\in A(p)$

$y_{a}\left(\pi_{a}^{p}-\eta_{a}^{p}+\varphi_{a}^{p}\right)=y_{a}$

$\forall p \in \hat{P}^{w}, w \in W, a \in A(p)$

$y_{a} \pi_{a}^{p} \geq 0$

$\forall p \in \hat{P}^{w}, w \in W, a \in A(p)$

$y_{a} \eta_{a}^{p} \geq 0$

$\forall p \in \hat{P}^{w}, w \in W, a \in A(p)$

$y_{a} \varphi_{a}^{p} \geq 0$

$\forall p \in \hat{P}^{w}, w \in W, a \in A(p)$

$\xi_{i}^{p} \geq 0$

$\forall p \in \hat{P}^{w}, w \in W, i \in N(p)$

$\varsigma_{i}^{p} \geq 0$

$\forall p \in \hat{P}^{w}, w \in W, i \in N(p)$

$y_{a}\left(-t_{a}+\gamma_{a}^{p}\right) \geq 0$

$\forall p \in \hat{P}^{w}, w \in W, a \in A(p)$

$y_{a}\left(\bar{t}_{a}-\gamma_{a}^{p}\right) \geq 0$

$\forall p \in \hat{P}^{w}, w \in W, a \in A(p)$

$y_{a}\left(-\lambda_{a}^{p}+\gamma_{a}^{p}\right) \geq 0$

$\forall p \in \hat{P}^{w}, w \in W, a \in A(p)$

$\left(1-y_{a}\right)\left(d_{a} \varpi-\beta_{i}^{p}+\beta_{j}^{p}\right)=0$

$\forall p \in \hat{P}^{w}, w \in W,(i, j)=a$

$\in A(p)$

$y_{a}\left(d_{a} \varpi-e \lambda_{a}^{p}-\beta_{i}^{p}+\beta_{j}^{p}\right)=0$

$\forall p \in \hat{P}^{w}, w \in W,(i, j)=a$

$\in A(p)$

$L_{\text {max }}-\beta_{i}^{p} \geq 0$

$\forall p \in \hat{P}^{w}, w \in W, i \in N(p)$

$-L_{o}+\beta_{o(w)}^{p}=0$

$\forall p \in \hat{P}^{w}, w \in W$

$-m^{w}+\beta_{i}^{p} \geq 0$

$\forall p \in \hat{P}^{w}, w \in W, i \in N(p)$ 


$$
\begin{array}{ll}
y_{a} \lambda_{a}^{p}\left(-\varphi_{a}^{p}-e \kappa_{a}^{p}\right)=0 & \forall p \in \hat{P}^{w}, w \in W, a \in A(p) \\
y_{a} \pi_{a}^{p}\left(-t_{a}+\gamma_{a}^{p}\right)=0 & \forall p \in \hat{P}^{w}, w \in W, a \in A(p) \\
y_{a} \eta_{a}^{p}\left(\bar{t}_{a}-\gamma_{a}^{p}\right)=0 & \forall p \in \hat{P}^{w}, w \in W, a \in A(p) \\
y_{a} \varphi_{a}^{p}\left(-\lambda_{a}^{p}+\gamma_{a}^{p}\right)=0 & \forall p \in \hat{P}^{w}, w \in W, a \in A(p) \\
\xi_{i}^{p}\left(L_{\max }-\beta_{i}^{p}\right)=0 & \forall p \in \hat{P}^{w}, w \in W, i \in N(p) \\
\left(-m^{w}+\beta_{i}^{p}\right) \varsigma_{i}^{p}=0 & \forall p \in \hat{P}^{w}, w \in W, i \in N(p) \\
\left(\sum_{a \in A(p)} t_{a}\left(1-y_{a}\right)+\sum_{a \in A(p)} \gamma_{a}^{p} y_{a}-\rho^{w}\right) f_{p}=0 & \forall p \in \hat{P}^{w}, w \in W \\
y_{a} \lambda_{a}^{p} \geq 0 &
\end{array}
$$

where $I$ is the given budget, $c_{a}$ is the cost for constructing one mile of charging lanes on link $a$, and $\rho^{w}$ is an auxiliary variable, representing the equilibrium trip time for O-D pair $w \in W$

In the above, the objective is to minimize the social cost or total travel time. The objective function can be extended to incorporate other considerations, e.g., maximizing the exposure of charging opportunities provided to EVs (e.g., Kuby et al., 2009). In this case, a weighted value of $\sum_{a \in A} v_{a} y_{a}$ can be added to the objective function.

Constraints (54) and (55) ensure that the total expense of charging-lane deployment must be within the given budget, and $y_{a}$ is a binary variable. The other constraints represent the optimality conditions of NE-VI as shown in Appendix B with the location variables of charging lanes.

\subsection{Solution algorithm}

As formulated, CLL is a mathematical problem with complementarity constraints, a class of problems difficult to solve especially for large problems (e.g., Luo et al., 1996). This paper applies an efficient active-set algorithm developed by Zhang et al. (2009) to solve CLL. The basic idea of the algorithm is first to solve a restricted version of CLL upon a pair of active sets corresponding to a particular deployment plan and obtain the multipliers corresponding to the active sets. Based on those multipliers, a binary knapsack problem is then constructed and solved to update the active sets. A new deployment plan is thus obtained, which improves the objective value. The deployment plan is updated iteratively until there is no better plan.

For a particular deployment plan, we define a pair of active sets $\Omega_{0}=\left\{a: y_{a}=0\right\}$ and $\Omega_{1}=\left\{a: y_{a}=1\right\}$. These two sets should be "complete", i.e., $\Omega_{0} \cup \Omega_{1}=A ; \Omega_{0} \cap \Omega_{1}=\emptyset$. Based on $\Omega_{0}$ and $\Omega_{1}$, the restricted version of CLL, i.e., RCLL, can be formulated below:

\section{RCLL:}

$$
\min _{\boldsymbol{\pi}, \boldsymbol{\eta}, \boldsymbol{\varphi}, \boldsymbol{\kappa}, \xi, \boldsymbol{\theta}, \boldsymbol{\zeta}, \boldsymbol{\beta}, \boldsymbol{\gamma}, \lambda, \boldsymbol{f}, \boldsymbol{v}, \boldsymbol{\rho}, \boldsymbol{y}} \sum_{w \in W} \rho^{w} g^{w}
$$


s.t. (27)-(29), (54), (56)-(82)

$\begin{array}{ll}y_{a}=0 & \forall a \in \Omega_{0} \\ y_{a}=1 & \forall a \in \Omega_{1}\end{array}$

Note that RCLL is another mathematical program with complementarity constraints, but its optimal solution can be easily obtained by solving NE-NLP upon the given deployment plan, i.e., $\left(\Omega_{0}, \Omega_{1}\right)$. The active-set procedure can be described as follows. For the proof of convergence, see Zhang et al. (2009).

Step 0: Set $\tau=1, \Omega_{0}^{1}=A$, and $\Omega_{1}^{1}=\emptyset$. Solve NE-NLP with an initial deployment plan $\left(\Omega_{0}^{1}, \Omega_{1}^{1}\right)$.

Step 1: Using the optimal solution of NE-NLP, construct a solution

$(\boldsymbol{\pi}, \boldsymbol{\eta}, \boldsymbol{\varphi}, \boldsymbol{\kappa}, \boldsymbol{\xi}, \boldsymbol{\theta}, \boldsymbol{\varsigma}, \boldsymbol{\beta}, \boldsymbol{\gamma}, \boldsymbol{\lambda}, \boldsymbol{f}, \boldsymbol{v}, \boldsymbol{\rho}, \boldsymbol{y})^{T}$ to RCLL with $\left(\Omega_{0}^{\tau}, \Omega_{1}^{\tau}\right)$. Then, solve RCLL again to determine $\lambda_{a}^{\tau}$ and $\mu_{a}^{\tau}$, the Lagrangian multipliers associated with constraints (83) and (84).

Set $T T^{\tau}=\sum_{w} \rho^{w} g^{w}$ and go to step 2 .

Step 2: Set $Q=-\infty$ and adjust the active sets by performing the following steps:

a) Let $(\widehat{\boldsymbol{k}}, \widehat{\boldsymbol{h}})$ solve the following knapsack problem:

$$
\min \sum_{a \in \Omega_{0}^{\tau}} \lambda_{a}^{\tau} k_{a}-\sum_{a \in \Omega_{1}^{\tau}} \mu_{a}^{\tau} h_{a}
$$

s.t.

$$
\begin{gathered}
\sum_{a \in \Omega_{0}^{\tau}} c_{a} d_{a} k_{a}-\sum_{a \in \Omega_{1}^{\tau}} c_{a} d_{a} h_{a} \leq I-\sum_{a \in \Omega_{1}^{\tau}} c_{a} d_{a} \\
\sum_{a \in \Omega_{0}^{\tau}} \lambda_{a}^{\tau} k_{a}-\sum_{a \in \Omega_{1}^{\tau}} \mu_{a}^{\tau} h_{a} \geq Q \\
k_{a}, h_{a} \in\{0,1\}, \forall a \in A
\end{gathered}
$$

If its optimal objective value is zero, stop and the current solution is optimal.

Otherwise, go to Step $2 b$.

b) Set:

i. $D=\sum_{a \in \Omega_{0}^{\tau}} \lambda_{a}^{\tau} \hat{k}_{a}-\sum_{a \in \Omega_{1}^{\tau}} \mu_{a}^{\tau} \hat{h}_{a}$,

ii. $\widehat{\Omega}_{0}=\left(\Omega_{0}^{\tau}-\left\{a \in \Omega_{0}^{\tau}: \hat{k}_{a}=1\right\}\right) \cup\left\{a \in \Omega_{1}^{\tau}: \hat{h}_{a}=1\right\}$,

iii. $\widehat{\Omega}_{1}=\left(\Omega_{1}^{\tau}-\left\{a \in \Omega_{1}^{\tau}: \hat{h}_{a}=1\right\}\right) \cup\left\{a \in \Omega_{0}^{\tau}: \hat{k}_{a}=1\right\}$.

c) Solve NE-NLP with a deployment plan vector $\widehat{\boldsymbol{y}}$ compatible with $\left(\widehat{\Omega}_{0}, \widehat{\Omega}_{1}\right)$. If its social cost $T T<T T^{\tau}$, go to Step $2 \mathrm{~d}$ since the location plan $\left(\widehat{\Omega}_{0}, \widehat{\Omega}_{1}\right)$ leads to a decrease in the social cost. Otherwise, set $Q=D+\varepsilon$, where $\varepsilon>0$ is sufficiently small, and return to Step 2 a. 
d) Set $\Omega_{0}^{\tau+1}=\widehat{\Omega}_{0}, \Omega_{1}^{\tau+1}=\widehat{\Omega}_{1}$, and $\tau=\tau+1$. Go to Step 1 .

At Step 0, for simplicity, the initial deployment plan can be constructing no charging lanes on the network. As previously mentioned, the optimal solution to NE-NLP may not be unique. At Step 0 and 2c, we can solve NE-NLP with different initial solutions, and select the one with the minimum social cost as the optimal solution.

At Step 2a, the objective of the knapsack problem is to minimize the total estimated changes to the social cost by adjusting the current plan. $k_{a}$ and $h_{a}$ are two sets of binary decision variables, where $k_{a}=1$ means shifting link $a$ from $\Omega_{0}$ to $\Omega_{1}$, and $h_{a}=1$ means the opposite. If the optimal value is zero, which implies that no better plan can be adjusted from the current plan to lead to a smaller social cost, then the algorithm will be terminated. Otherwise, an adjustment plan $\widehat{\boldsymbol{y}}$ is obtained in Step 2b. However, because the Lagrangian multipliers are only estimates of the changes, $\widehat{\boldsymbol{y}}$ may not lead to an actual smaller social cost. Thus, Step 2c is to verify whether $\widehat{\boldsymbol{y}}$ is a better plan vector or not. If $\widehat{\boldsymbol{y}}$ doesn't yield a smaller social cost, then a new adjustment plan is obtained by solving the knapsack problem at Step $2 \mathrm{a}$ with a slight increased $Q$ by $\varepsilon$.

\subsection{Numerical examples}

In this section, we first solve CLL for the Nguyent-Dupius network with various budgets to examine the active-set algorithm. Consider that $c_{a}=1 \mathrm{unit} / \mathrm{mi}, \forall a \in A$ and the budget is 40 unit. The algorithm yields a deployment plan of locating charging lanes on Link 1-5 and 4-9, with a social cost of 152,159 min. Fig. 7 enumerates all 220 feasible deployment plans and verifies that the active-set algorithm generates a global optimum solution. We further increase the budget to be $50,60,70,80$, and 90 respectively and the active-set algorithm also produces global optimum solution in all these tests.

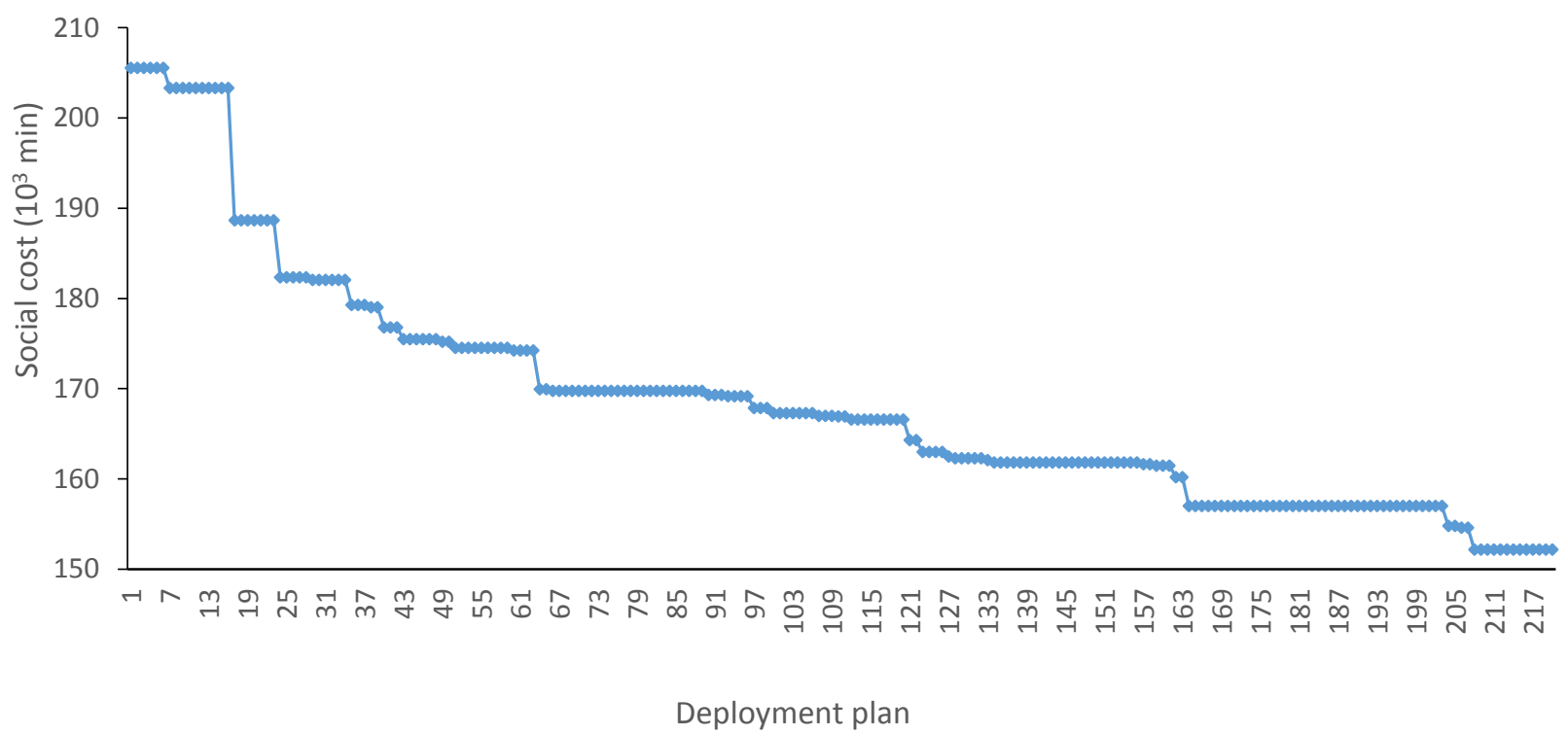

Fig. 7 Enumeration of all deployment plans 
We then further solve CLL for the Sioux Falls network, which consists of 76 links, and 24 nodes (see, e.g., He et al, 2014). Note that the original Sioux Falls network primarily consists of urban arterials where charging lanes may not be needed. This numerical example only utilizes the topology of the Sioux Falls network while the original link characteristics are revised to reflect a regional freeway network. Specifically, the link performance functions are BPR function and the link characteristics are shown in Table 7, and the O-D demands can be found in Table 8 . In addition, we set $L_{0}=20 \mathrm{kWh}, L_{\max }=24 \mathrm{kWh}, e=1.5 \mathrm{kWh} / \mathrm{min}, \varpi=0.29$ $\mathrm{kWh} / \mathrm{mi}$, and the minimum speed limit is set to be $30 \mathrm{mph}$. The budget is set as 1600 units, and $c_{a}$ is assumed to be one thousandth of the link capacity. Fig. 8 shows how the social cost decreases at each iteration, i.e., each outer loop of the active-set algorithm. The algorithm terminates at the $6^{\text {th }}$ iteration, and produces a deployment plan that locate charging lanes on links $9-5,10-11,13-12,13-24$, and 21-20. The plan reduces the social cost by $13.23 \%$. 
Table 7 Sioux Falls network characteristics

\begin{tabular}{|c|c|c|c|c|c|c|c|}
\hline Link & $\begin{array}{l}\text { Free-flow } \\
\text { travel time } \\
\quad(\min )\end{array}$ & $\begin{array}{c}\text { Cap } \\
\left(\times 10^{3}\right)\end{array}$ & $\begin{array}{c}\text { Length } \\
\text { (mi) }\end{array}$ & Link & $\begin{array}{l}\text { Free-flow } \\
\text { travel time } \\
\text { (min) }\end{array}$ & $\begin{array}{c}\text { Cap } \\
\left(\times 10^{3}\right)\end{array}$ & $\begin{array}{l}\text { Length } \\
\text { (mi) }\end{array}$ \\
\hline $1-2$ & 5.66 & 6.02 & 7.56 & $13-24$ & 11.33 & 10.18 & 15.12 \\
\hline $1-3$ & 11.33 & 9.01 & 15.12 & $14-11$ & 11.33 & 9.75 & 15.12 \\
\hline $2-1$ & 5.66 & 12.02 & 7.56 & $14-15$ & 14.16 & 10.26 & 18.90 \\
\hline $2-6$ & 14.16 & 15.92 & 18.90 & $14-23$ & 11.33 & 9.85 & 15.12 \\
\hline $3-1$ & 11.33 & 46.81 & 15.12 & $15-10$ & 16.99 & 27.02 & 22.68 \\
\hline $3-4$ & 4.72 & 34.22 & 6.30 & $15-14$ & 14.16 & 10.26 & 18.90 \\
\hline $3-12$ & 11.33 & 46.81 & 15.12 & $15-19$ & 11.33 & 9.64 & 15.12 \\
\hline $4-3$ & 4.72 & 25.82 & 6.30 & $15-22$ & 11.33 & 20.63 & 15.12 \\
\hline $4-5$ & 5.66 & 28.25 & 7.56 & $16-8$ & 14.16 & 10.09 & 18.90 \\
\hline $4-11$ & 16.99 & 9.04 & 22.68 & $16-10$ & 14.16 & 10.27 & 18.90 \\
\hline $5-4$ & 5.66 & 46.85 & 7.56 & $16-17$ & 5.66 & 10.46 & 7.56 \\
\hline $5-6$ & 11.33 & 13.86 & 15.12 & $16-18$ & 8.50 & 39.36 & 11.34 \\
\hline 5-9 & 14.16 & 10.52 & 18.90 & $17-10$ & 19.82 & 9.99 & 26.46 \\
\hline $6-2$ & 14.16 & 9.92 & 18.90 & $17-16$ & 5.66 & 10.46 & 7.56 \\
\hline $6-5$ & 11.33 & 9.90 & 15.12 & $17-19$ & 5.66 & 9.65 & 7.56 \\
\hline $6-8$ & 5.66 & 21.62 & 7.56 & $18-7$ & 5.66 & 46.81 & 7.56 \\
\hline $7-8$ & 8.50 & 15.68 & 11.34 & $18-16$ & 8.50 & 39.36 & 11.34 \\
\hline $7-18$ & 5.66 & 46.81 & 7.56 & $18-20$ & 11.33 & 8.11 & 15.12 \\
\hline 8-6 & 5.66 & 9.80 & 7.56 & $19-15$ & 11.33 & 4.42 & 15.12 \\
\hline $8-7$ & 8.50 & 15.68 & 11.34 & $19-17$ & 5.66 & 9.65 & 7.56 \\
\hline $8-9$ & 9.44 & 10.10 & 12.60 & $19-20$ & 11.33 & 10.01 & 15.12 \\
\hline $8-16$ & 14.16 & 10.09 & 18.90 & $20-18$ & 11.33 & 8.11 & 15.12 \\
\hline $9-5$ & 14.16 & 20.00 & 18.90 & $20-19$ & 11.33 & 6.05 & 15.12 \\
\hline $9-8$ & 9.44 & 10.10 & 12.60 & $20-21$ & 5.66 & 10.12 & 7.56 \\
\hline $9-10$ & 8.50 & 27.83 & 11.34 & $20-22$ & 14.16 & 10.15 & 18.90 \\
\hline $10-9$ & 8.50 & 27.83 & 11.34 & $21-20$ & 5.66 & 10.12 & 7.56 \\
\hline $10-11$ & 14.16 & 20.00 & 18.90 & $21-22$ & 5.66 & 10.46 & 7.56 \\
\hline $10-15$ & 16.99 & 27.02 & 22.68 & $21-24$ & 8.50 & 9.77 & 11.34 \\
\hline $10-16$ & 14.16 & 10.27 & 18.90 & $22-15$ & 11.33 & 20.63 & 15.12 \\
\hline $10-17$ & 19.82 & 9.99 & 26.46 & $22-20$ & 14.16 & 10.15 & 18.90 \\
\hline $11-4$ & 16.99 & 9.82 & 22.68 & $22-21$ & 5.66 & 10.46 & 7.56 \\
\hline $11-10$ & 14.16 & 20.00 & 18.90 & $22-23$ & 11.33 & 10 & 15.12 \\
\hline $11-12$ & 16.99 & 9.82 & 22.68 & $23-14$ & 11.33 & 9.85 & 15.12 \\
\hline $11-14$ & 11.33 & 9.75 & 15.12 & $23-22$ & 11.33 & 10 & 15.12 \\
\hline $12-3$ & 11.33 & 46.81 & 15.12 & $23-24$ & 5.66 & 10.16 & 7.56 \\
\hline $12-11$ & 16.99 & 9.82 & 22.68 & $24-13$ & 11.33 & 11.38 & 15.12 \\
\hline $12-13$ & 8.50 & 51.80 & 11.34 & $24-21$ & 8.50 & 9.77 & 11.34 \\
\hline $13-12$ & 8.50 & 51.80 & 11.34 & $24-23$ & 5.66 & 10.16 & 7.56 \\
\hline
\end{tabular}


Table 8 O-D demand of Sioux Falls network

\begin{tabular}{r|rrrrrrrrrr}
\hline OlD & 1 & 2 & 4 & 5 & 7 & 13 & 18 & 20 & 21 & 24 \\
\hline 1 & 0 & 500 & 2500 & 1000 & 2500 & 2500 & 500 & 1500 & 500 & 500 \\
2 & 500 & 0 & 1000 & 500 & 1000 & 1500 & 1000 & 500 & 1000 & 1000 \\
4 & 2500 & 1000 & 0 & 2500 & 2000 & 3000 & 500 & 1500 & 1000 & 1000 \\
5 & 1000 & 500 & 2500 & 0 & 1000 & 1000 & 1000 & 500 & 500 & 1000 \\
7 & 2500 & 1000 & 2000 & 1000 & 0 & 2000 & 1000 & 2500 & 1000 & 500 \\
13 & 2500 & 1500 & 3000 & 1000 & 2000 & 0 & 500 & 3000 & 3000 & 4000 \\
18 & 500 & 1000 & 500 & 1000 & 1000 & 500 & 0 & 2000 & 500 & 1000 \\
20 & 1500 & 500 & 1500 & 500 & 2500 & 3000 & 2000 & 0 & 6000 & 2000 \\
21 & 500 & 1000 & 1000 & 500 & 1000 & 3000 & 500 & 6000 & 0 & 2500 \\
24 & 500 & 1000 & 1000 & 1000 & 500 & 3500 & 1000 & 2000 & 2500 & 0 \\
\hline
\end{tabular}

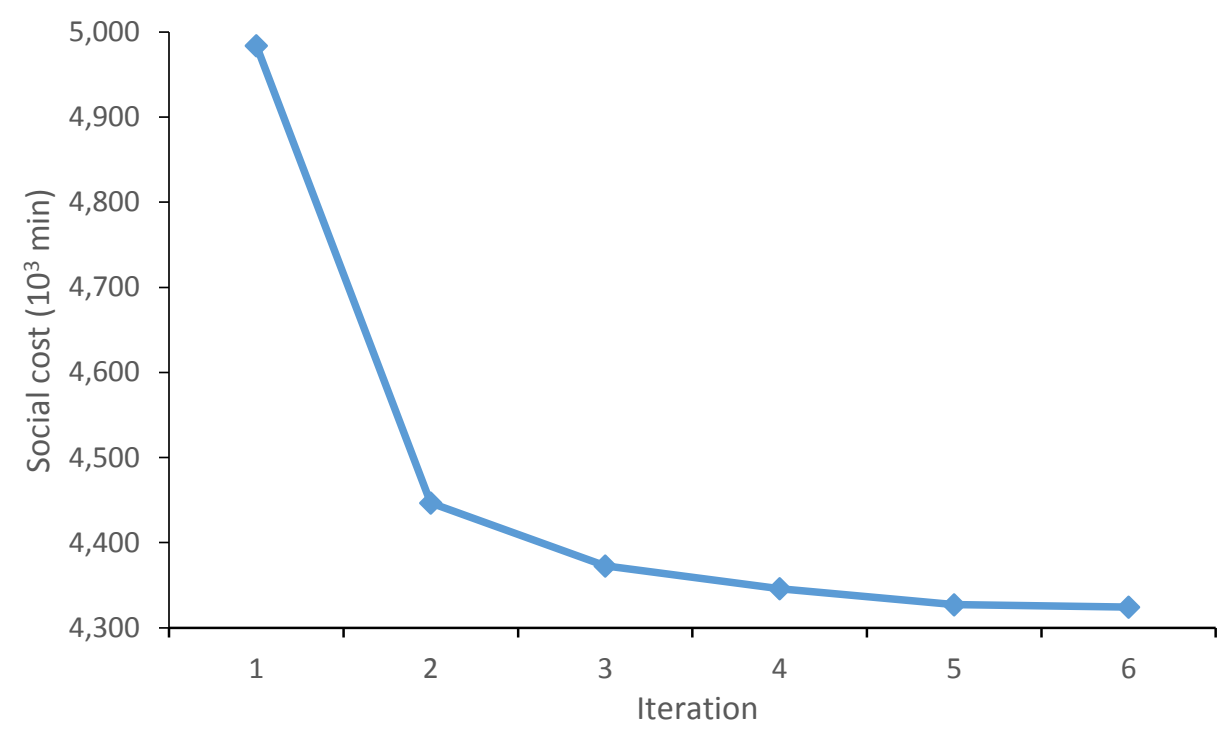

Fig. 8 Convergence of the active-set algorithm

\section{Conclusion}

We have investigated a mathematical approach to optimize the deployment of charging lanes in a general network. Given that remarkable progress has been made in the field of CWD, charging lanes can be deployed to allow EV drivers to charge their vehicles when traveling from their origins to destinations. The drivers are assumed to minimize their trip times by selecting their paths and recharging plans. A linear program is formulated to describe their decision of recharging plans and their subsequent route choices yield a network equilibrium state, which is formulated as a VI problem. An iterative procedure is developed to solve the VI problem efficiently. Numerical examples are presented to demonstrate the network equilibrium model. It 
is observed that drivers may slow down so that their batteries can be sufficiently recharged to cover their trips. With the established network equilibrium conditions, we have formulated a deployment model that optimizes the locations of charging lanes for a given budget. The model is a mathematical program with complementarity constraints and is solved by an active-set algorithm. Numerical examples based on the Nguyent-Dupius and Sioux Falls networks demonstrate the effectiveness of the active-set algorithm and the use of the optimal deployment model.

Our future research will consider the simultaneous deployment of both dynamic and static charging infrastructure, which affects drivers' travel choices in a different manner. They also likely offer different prices of electricity and charging efficiency, attracting different types of users. It would be interesting to investigate how to optimally deploy these charging infrastructures considering heterogeneous drivers.

\section{Acknowledgements}

The research is partially supported by grants from the National Science Foundation (CNS1239364; CMMI-1362631; EFRI-1441231) and National Natural Science Foundation of China (71501107). We would also like to thank the support from Lloyd's Register Foundation (LRF). LRF helps to protect life and property by supporting engineering-related education, public engagement and the application of research.

\section{References}

Aghassi, M., Bertsimas, D., Perakis, G., 2006. Solving asymmetric variational inequalities via convex optimization. Operations Research Letters 34 (5), 481-490.

Bansal, P., 2015. Charging of electric vehicles: technology and policy implications. Journal of Science Policy and Governance 6 (1).

Choi, S., Huh, J., Lee, W. Y., Lee, S. W., Rim, C. T., 2013. New cross-segmented power supply rails for roadway-powered electric vehicles. Power Electronics, IEEE Transactions on 28 (12), 5832-5841.

Cirimele, V., Freschi, F., Guglielmi, P., 2014. Wireless power transfer structure design for electric vehicle in charge while driving. In Electrical Machines (ICEM), 2014 International Conference on. IEEE, 2461-2467.

Dong, J., Liu, C., Lin, Z., 2014. Charging infrastructure planning for promoting battery electric vehicles: an activity-based approach using multiday travel data. Transportation Research Part C 38, 44-55.

Drud, A., 1994. CONOPT - a large scale GRG code. ORSA Journal on Computing 6 (2), 207 216.

Electric Power Research Institute (EPRI), 2007. Environmental assessment of plug-in hybrid electric vehicles. Volume 1: Nationwide Greenhouse Gas Emissions. http://www.epri.com/abstracts/pages/productabstract.aspx?ProductId=000000000001015 $\underline{325}$ (accessed April 07, 2015).

Fontana, M.W., 2013. Optimal routes for electric vehicles facing uncertainty, congestion, and energy constraints. PhD Dissertation. Massachusetts Institute of Technology. 
Fuller, M., 2016. Wireless charging in California: Range, recharge, and vehicle electrification. Transportation Research Part C 67, 343-356.

Geier, B., 2015. Electric vehicle sales charged up in 2014. http://fortune.com/2015/01/08/electric-vehicle-sales-2014/ (accessed March 24, 2015).

Harker, P.T., Pang, J.-S., 1990. Finite-dimensional variational inequality and nonlinear complementarity problems: a survey of theory, algorithms and applications. Mathematical Programming 48, 161-220.

He, F., Wu, D., Yin, Y., Guan, Y., 2013a. Optimal deployment of public charging stations for plug-in hybrid electric vehicles. Transportation Research Part B 47 (1), 87-101.

He, F., Yin, Y., Zhou, J., 2013b. Integrated pricing of roads and electricity enabled by wireless power transfer. Transportation Research Part C 34, 1-15.

He, F., Yin, Y., Lawphongpanich, S., 2014. Network equilibrium models with battery electric vehicles. Transportation Research Part B 67, 306-319.

He, F., Yin, Y., Zhou, J., 2015. Deploying public charging stations for electric vehicles on urban road networks. Transportation Research Part C 60, 227-240.

He, F., Yin, Y., Wang, J., Yang, Y., 2016. Sustainability SI: optimal prices of electricity at public charging stations for plug-in electric vehicles. Network and Spatial Economics 16 (1), 131-154.

Herron, D., 2014. Siemens eHighway of the future concept. http://greentransportation.info/evcharging/electric-roads/siemens-ehighway-future.html (accessed March 24, 2015).

Highways England, 2015a. Feasibility study: Power electric vehicles on England's major roads. http://www.highways.gov.uk/knowledge/publications/1902/ (accessed September 15, 2015).

Highways England, 2015b. Off road trials for "electric highways" technology. https://www.gov.uk/government/news/off-road-trials-for-electric-highways-technology (accessed September 15, 2015).

Jiang, N., Xie, C., Waller, T., 2012. Path-constrained traffic assignment: model and algorithm. Transportation Research Record 2283, 25-33.

Jiang, N., Xie, C., Duthie, J. C., Waller, S. T., 2014. A network equilibrium analysis on destination, route and parking choices with mixed gasoline and electric vehicular flows. European Journal on Transportation and Logistics 3, 55-92.

Jung, J., Chow, J. Y., Jayakrishnan, R., Park, J. Y., 2014. Stochastic dynamic itinerary interception refueling location problem with queue delay for electric taxi charging stations. Transportation Research Part C 40, 123-142.

Kang, J. E., Recker, W. W., 2014. Strategic hydrogen refueling station locations with scheduling and routing considerations of individual vehicles. Transportation Science 49 (4), 767-783.

Kuby, M., Lines, L., Schultz, R., Xie, Z., Kim, J.-G., Lim, S., 2009. Optimization of hydrogen stations in Florida using the flow-refueling location model. International Journal of Hydrogen Energy 34, 6045-6064

Lukic, S., Pantic, Z., 2013. Cutting the cord: Static and dynamic inductive wireless charging of electric vehicles. Electrification Magazine, IEEE 1 (1), 57-64.

Luo, Z.-Q., Pang, J.-S., Ralph, D., 1996. Mathematical programs with equilibrium constraints. Cambridge University Press, New York, New York.

Morris, C., 2015. Utah State University builds a dynamic wireless charging test track. http://chargedevs.com/features/utah-state-university-builds-a-dynamic-wireless-chargingtest-track/ (accessed March 24, 2015). 
Morrow, K., Karner, D., Francfort, J., 2008. Plug-in hybrid electric vehicle charging infrastructure review. Final Report to US Department of Energy Vehicle Technologies Program - Advanced Vehicle Testing Activity.

Nguyen, S., Dupuis, C., 1984. An efficient method for computing traffic equilibria in networks with asymmetric transportation costs. Transportation Science 18 (2), 185-202.

Nie, Y. M., Ghamami, M., 2013. A corridor-centric approach to planning electric vehicle charging infrastructure. Transportation Research Part B 57, 172-190.

Ning, P., Miller, J. M., Onar, O. C., White, C. P., 2013. A compact wireless charging system for electric vehicles. In Energy Conversion Congress and Exposition (ECCE), IEEE, 36293634.

Nissan USA, 2013. http://www.nissanusa.com/electric-cars/leaf/charging-range/battery/ (accessed March 24, 2015).

Scania Newsroom, 2014. Scania tests next-generation electric vehicles. http://newsroom.scania.com/en-group/2014/03/13/scania-tests-next-generation-electricvehicles/ (accessed March 24, 2015).

Schiller, B., 2013. Volvo tests a road that can charge cars and trucks. http://www.fastcoexist.com/3016069/futurist-forum/volvo-tests-a-road-that-can-chargecars-and-trucks (accessed March 24, 2015).

Shinohara, N., Kubo, Y., Tonomura, H., 2013. Wireless charging for electric vehicle with microwaves. In Electric Drives Production Conference (EDPC), IEEE, 1-4.

Siemens, 2014. Siemens tests eHighway system in California. http://w1.siemens.com.cn/news_en/news_articles_en/2669.aspx (accessed June 04, 2015)

Riemann, R., Wang, D. Z., Busch, F., 2015. Optimal location of wireless charging facilities for electric vehicles: flow-capturing location model with stochastic user equilibrium. Transportation Research Part C 58, 1-12.

TRL, 2015. Feasibility of using dynamic wireless power transfer on the strategic road network for EV take-up. http://www.trl.co.uk/news-hub/trl-press-releases/2015/march/feasibilityof-using-dynamic-wireless-power-transfer-on-the-strategic-road-network-for-ev-take-up/ (accessed June 05, 2015).

US Department of Energy, 2013. http://www.fueleconomy.gov/feg/evsbs.shtml (accessed March 24, 2015).

Vilathgamuwa, D. M., Sampath, J. P. K., 2015. Wireless power transfer (WPT) for electric vehicles (EVs) - present and future trends. Plug In Electric Vehicles in Smart Grids. Springer Singapore, 33-60.

Wu, X., Freese, D., Cabrera, A., Kitch, W. A., 2015. Electric vehicles' energy consumption measurement and estimation. Transportation Research Part D 34, 52-67.

Yang, H., Ye, H., Li, X., Zhao, B., 2015. Speed limits, speed selection and network equilibrium. Transportation Research Part C 51, 260-273.

Yu, X., Sandhu, S., Beiker, S., Sassoon, R., Fan, S., 2011. Wireless energy transfer with the presence of metallic planes. Applied Physics Letters 99 (21), 214102.

Zhang, L., Lawphongpanich, S., Yin, Y., 2009. An active-set algorithm for discrete network design problems. Transportation and Traffic Theory 2009: Golden Jubilee 2009, 283-300. 


\section{Appendix A}

This appendix extends the proposed model by considering that the prevailing speed (travel time) on a charging link not only depends on the congestion level, but also is affected by the average travel speed (time). For completeness and convenience, we repeat some previously presented constraints as well as parameters here.

With a given traffic flow distribution $\widetilde{\boldsymbol{v}}$, a given path flow pattern $\tilde{\boldsymbol{f}}$, and given actual travel times on charging links along the other paths $\tilde{S}_{a}^{\hat{p}}, \forall a \in \hat{A}, \hat{p} \in \hat{P} \backslash p$, the optimal decision of a driver traveling along path $p \in \hat{P}^{w}$ can be obtained by solving the following extended recharging time (ERT) problem whose decision variables include recharging time, actual travel time and the SOC of the battery:

ERT:

$$
\begin{array}{ll}
\min _{L, \hat{s}, s^{p}} \sum_{a \in \hat{A}(p)} s_{a}^{p}+\sum_{a \in A(p) \backslash \hat{A}(p)} t_{a}\left(\tilde{v}_{a}\right) \\
s_{a}^{p} \geq t_{a}\left(\tilde{v}_{a}\right) & \forall a \in \hat{A}(p) \\
s_{a}^{p} \leq \bar{t}_{a} & \forall a \in \hat{A}(p) \\
\hat{s}_{a}^{p} \leq s_{a}^{p} & \forall a \in \hat{A}(p) \\
L_{j}^{p}-L_{i}^{p}+d_{a} \varpi=0 & \forall(i, j)=a \in A(p) \backslash \hat{A}(p) \\
L_{j}^{p}-L_{i}^{p}+d_{a} \varpi-\hat{s}_{a}^{p} e=0 & \forall(i, j)=a \in \hat{A}(p) \\
L_{i}^{p} \leq L_{\max } & \forall i \in N(p) \\
L_{o(w)}^{p}=L_{0} & \\
\hat{s}_{a}^{p} \geq 0 & \forall a \in \hat{A}(p) \\
L_{i}^{p} \geq m^{w} & \forall i \in N(p) \\
s_{a}^{p} \tilde{v}_{a} \geq \tilde{\beta} \sum_{w \in W} \sum_{\hat{p} \in \hat{P}^{w}} \tilde{s}_{a}^{\hat{p}} \tilde{f}_{\hat{p}} \delta_{a}^{\hat{p}} & \forall a \in \hat{A}(p)
\end{array}
$$

where $\tilde{\beta}$ is a coefficient with a range $[0,1]$.

Different from the RT program, constraint (A.10) is newly added. Specifically, constraint (A.10) ensures that if $\tilde{v}_{a}>0$, then $s_{a}^{p} \geq \tilde{\beta} \frac{\sum_{w \in W} \sum_{\widehat{p} \in \widehat{P} w} \tilde{s}_{a}^{\hat{p}} \tilde{f}_{\hat{p}} \delta_{a}^{\hat{p}}}{\tilde{v}_{a}}$. That is, the actual travel time $s_{a}^{p}$ must be no less than the average actual travel time on charging link $a$ times a coefficient $\tilde{\beta}$. Obviously, $\tilde{\beta}$ is a nonnegative parameter between 0 to 1 . Specifically, if $\tilde{\beta}=1$, actual travel times of all drivers on the charging link are the same. Note that $\widetilde{\beta}$ could be further endogenized to capture the travel speed dispersion on a particular link. We leave it to future research.

ERT is a linear program written for each usable path $p \in \hat{P}^{w}, w \in W$. Below are its optimality conditions:
(A.1)-(A.10) 


$$
\begin{aligned}
& -\hat{\kappa}_{i j}^{p}+\hat{\kappa}_{k i}^{p}+\hat{\xi}_{i}^{p}-\hat{\beta}_{i}^{p}=0 \quad \forall(i, j),(k, i)=a \in A(p), \\
& -\hat{\kappa}_{o(w) j}^{p}+\hat{\xi}_{o(w)}^{p}+\hat{\theta}_{o(w)}^{p}-\hat{\beta}_{o(w)}^{p}=0 \quad \forall(o(w), j)=a \in A(p) \\
& \hat{\kappa}_{k d(w)}^{p}+\hat{\xi}_{d(w)}^{p}-\hat{\beta}_{d(w)}^{p}=0 \quad \forall(k, d(w))=a \in A(p) \\
& \hat{\varphi}_{a}^{p}-e \hat{\kappa}_{a}^{p}-\hat{\alpha}_{a}^{p}=0 \quad \forall a \in \hat{A}(p) \\
& 1-\hat{\pi}_{a}^{p}+\hat{\eta}_{a}^{p}-\hat{\varphi}_{a}^{p}-\left(\tilde{v}_{a}-\tilde{\beta} \tilde{f}_{p}\right) \hat{\vartheta}_{a}^{p}=0 \quad \forall a \in \hat{A}(p) \\
& \left(s_{a}^{p}-t_{a}\left(v_{a}\right)\right) \hat{\pi}_{a}^{p}=0 \quad \forall a \in \hat{A}(p) \\
& \left(s_{a}^{p}-\bar{t}_{a}\right) \hat{\eta}_{a}^{p}=0 \quad \forall a \in \hat{A}(p) \\
& \left(\hat{s}_{a}^{p}-s_{a}^{p}\right) \hat{\varphi}_{a}^{p}=0 \quad \forall a \in \hat{A}(p) \\
& \left(L_{i}^{p}-L_{\text {max }}\right) \hat{\xi}_{i}^{p}=0 \quad \forall i \in N(p) \\
& \hat{s}_{a}^{p} \hat{\alpha}_{a}^{p}=0 \quad \forall a \in \hat{A}(p) \\
& \left(L_{i}^{p}-m^{w}\right) \hat{\beta}_{i}^{p}=0 \quad \forall i \in N(p) \\
& \hat{\pi}_{a}^{p} \geq 0 \quad \forall a \in \hat{A}(p) \\
& \hat{\eta}_{a}^{p} \geq 0 \quad \forall a \in \hat{A}(p) \\
& \hat{\varphi}_{a}^{p} \geq 0 \quad \forall a \in \hat{A}(p) \\
& \hat{\xi}_{i}^{p} \geq 0 \quad \forall i \in N(p) \\
& \widehat{\alpha}_{a}^{p} \geq 0 \quad \forall a \in \hat{A}(p) \\
& \hat{\beta}_{i}^{p} \geq 0 \quad \forall i \in N(p) \\
& \left(s_{a}^{p} \tilde{v}_{a}-\tilde{\beta} \sum_{w \in W} \sum_{\hat{p} \in \hat{P} W} \tilde{s}_{a}^{\hat{p}} \tilde{\hat{f}}_{\hat{p}} \delta_{a}^{\hat{p}}\right) \vartheta_{a}^{p}=0 \quad \forall a \in \hat{A}(p) \\
& \vartheta_{a}^{p} \geq 0 \quad \forall a \in \hat{A}(p)
\end{aligned}
$$

where $\hat{\pi}_{a}^{p}, \hat{\eta}_{a}^{p}, \hat{\varphi}_{a}^{p}, \hat{\xi}_{i}^{p}, \bar{\theta}_{o(w)}^{p}, \hat{\alpha}_{a}^{p}, \hat{\beta}_{i}^{p}$, and $\hat{\vartheta}_{a}^{p}$ are the multipliers associated with constraints (A.1), (A.2), (A.3), (A.6), (A.7), (A.8), (A.9), and (A.10), while $\hat{\kappa}_{a}^{p}$ is the multiplier associated with (A.4) and (A.5).

Mathematically, we can define the network equilibrium conditions as follows:

(A.1)-(A.29)

$$
\begin{array}{ll}
\sum_{p \in \hat{P}^{w}} f_{p}=g^{w} & \forall w \in W \\
v_{a}=\sum_{w \in W} \sum_{p \in \widehat{P}^{w}} f_{p} \delta_{a}^{p} & \forall a \in A
\end{array}
$$




$$
\begin{array}{ll}
f_{p} \geq 0 & \forall p \in \hat{P}^{w}, w \in W \\
\left(\sum_{a \in A(p) \backslash \hat{A}(p)} t_{a}\left(v_{a}\right)+\sum_{a \in \hat{A}(p)} s_{a}^{p}-\mu^{w}\right) f_{p}=0 & \forall p \in \hat{P}^{w}, w \in W \\
\sum_{a \in A(p) \backslash \hat{A}(p)} t_{a}\left(v_{a}\right)+\sum_{a \in \hat{A}(p)} s_{a}^{p} \geq \mu^{w} & \forall p \in \hat{P}^{w}, w \in W
\end{array}
$$

To formulate an equivalent network equilibrium model, we first define a set $\bar{\Lambda}=$ $\{(\boldsymbol{L}, \widehat{\boldsymbol{s}}, \boldsymbol{s}, \widehat{\boldsymbol{\pi}}, \widehat{\boldsymbol{\vartheta}}, \boldsymbol{f}, \boldsymbol{v})\}$, where the vector satisfies conditions (A.2)-(A.9), (A.22), and (A.29)-(A.32).

The network equilibrium conditions (A.1)-(A.34) are equivalent to finding $\left(\boldsymbol{L}^{*}, \hat{\boldsymbol{s}}^{*}, \boldsymbol{s}^{*}, \widehat{\boldsymbol{\pi}}^{*}, \widehat{\boldsymbol{\vartheta}}^{*}, \boldsymbol{f}^{*}, \boldsymbol{v}^{*}\right) \in \bar{\Lambda}$ that solves the following variational inequality:

ENE-VI:

$$
\begin{aligned}
& \sum_{p \in \hat{P}^{W}, w \in W} \sum_{a \in \hat{A}(p)} {\left[\left(1-\hat{\pi}_{a}^{p^{*}}-\left(v_{a}^{*}-\tilde{\beta} f_{p}^{*}\right) \vartheta_{a}^{p^{*}}\right)\left(s_{a}^{p}-s_{a}^{p^{*}}\right)+\left(s_{a}^{p^{*}}-t_{a}\left(v_{a}^{*}\right)\right)\left(\hat{\pi}_{a}^{p}-\hat{\pi}_{a}^{p^{*}}\right)\right.} \\
&\left.+\left(s_{a}^{p^{*}} v_{a}^{*}-\tilde{\beta} \sum_{\hat{p} \in \hat{P} \widehat{w}, \hat{w} \in \widehat{W}} s_{a}^{\hat{p}^{*}} f_{\hat{p}}^{*} \delta_{a}^{\hat{p}}\right)\left(\vartheta_{a}^{p}-\vartheta_{a}^{p^{*}}\right)\right] \\
&+\sum_{p \in \widehat{P}^{w}, w \in W}\left[\sum_{a \in \hat{A}(p)} s_{a}^{p^{*}}\left(v_{a}-v_{a}^{*}\right)+\sum_{a \in A(p) \backslash \hat{A}(p)} t_{a}\left(v_{a}^{*}\right)\left(v_{a}-v_{a}^{*}\right)\right] \\
& \geq 0, \forall(\boldsymbol{L}, \hat{\boldsymbol{s}}, \boldsymbol{s}, \widehat{\boldsymbol{\pi}}, \widehat{\boldsymbol{\vartheta}}, \boldsymbol{f}, \boldsymbol{v}) \in \bar{\Lambda}
\end{aligned}
$$

The equivalence can be established by expressing the optimality conditions of ENE-VI and comparing them with the network equilibrium conditions. The solution procedure for ENEVI is similar to the one for NE-VI.

\section{Appendix B}

This appendix includes the proof of proposition 2. The optimality conditions of NE-VI can be written as follows: 


$$
\begin{aligned}
& \sum_{a \in \hat{A} \backslash \hat{A}(p)} t_{a}+\sum_{a \in \hat{A}(p)} t_{a} \pi_{a}^{p}-\sum_{a \in \hat{A}(p)} \bar{t}_{a} \eta_{a}^{p}-\sum_{a \in A(p)} d_{a} \varpi \kappa_{a}^{p} \\
& -\sum_{i \in N(p)} L_{\max } \xi_{i}^{p}+L_{o} \theta_{o(w)}^{p} \quad \forall p \in \hat{P}^{w}, w \in W \\
& +\sum_{i \in N(p)} m^{w} \varsigma_{i}^{p}-\alpha_{p}-\rho^{w}=0 \\
& -t_{a}+\gamma_{a}^{p}-\mu_{a}^{\pi, p}=0 \\
& \bar{t}_{a}-\gamma_{a}^{p}-\mu_{a}^{\eta, p}=0 \\
& -\lambda_{a}^{p}+\gamma_{a}^{p}-\mu_{a}^{\varphi, p}=0 \\
& d_{a} \varpi-\beta_{i}^{p}+\beta_{j}^{p}=0 \\
& d_{a} \varpi-e \lambda_{a}^{p}-\beta_{i}^{p}+\beta_{j}^{p}=0 \\
& L_{\max }-\beta_{i}^{p}-\mu_{i}^{\xi, p}=0 \\
& -L_{o}+\beta_{o(w)}^{p}=0 \\
& -m^{w}+\beta_{i}^{p}-\mu_{i}^{\zeta, p}=0 \\
& \left(-\varphi_{a}^{p}-e \kappa_{a}^{p}\right) \lambda_{a}^{p}=0 \\
& \pi_{a}^{p} \mu_{a}^{\pi, p}=0 \\
& \eta_{a}^{p} \mu_{a}^{\eta, p}=0 \\
& \varphi_{a}^{p} \mu_{a}^{\varphi, p}=0 \\
& \xi_{i}^{p} \mu_{i}^{\xi, p}=0 \\
& \varsigma_{i}^{p} \mu_{i}^{\varsigma, p}=0 \\
& f_{p} \alpha_{p}=0 \\
& \lambda_{a}^{p} \geq 0 \\
& \mu_{a}^{\pi, p} \geq 0 \\
& \mu_{a}^{\eta, p} \geq 0 \\
& \mu_{a}^{\varphi, p} \geq 0 \\
& \mu_{i}^{\xi, p} \geq 0 \\
& \mu_{i}^{s, p} \geq 0 \\
& \alpha_{p} \geq 0
\end{aligned}
$$

$(\boldsymbol{\pi}, \boldsymbol{\eta}, \boldsymbol{\varphi}, \boldsymbol{\kappa}, \boldsymbol{\xi}, \boldsymbol{\theta}, \boldsymbol{s}, \boldsymbol{f}, \boldsymbol{v}) \in \Lambda$

where $\rho^{w}, \alpha_{p}, \lambda_{a}^{p}, \gamma_{a}^{p}, \mu_{a}^{\pi, p}, \mu_{a}^{\eta, p}, \mu_{a}^{\varphi, p}, \mu_{i}^{\xi, p}$, and $\mu_{i}^{\zeta, p}$ are the multipliers of constraints (27), (29), (32), (36), (37), (38), (39), (40), and (41); $\beta_{i}^{p}$ is the multiplier of (33), (34) and (35).

First of all, we prove the equality below: 


$$
\begin{aligned}
\sum_{a \in \widehat{A}(p)} t_{a} \pi_{a}^{p}- & \sum_{a \in \widehat{A}(p)} \bar{t}_{a} \eta_{a}^{p}-\sum_{a \in A(p)} d_{a} \varpi \kappa_{a}^{p}-\sum_{i \in N(p)} L_{m a x} \xi_{i}^{p}+L_{o(w)} \theta_{o(w)}^{p}+\sum_{i \in N(p)} m^{w} s_{i}^{p} \\
& =\sum_{a \in \hat{A}(p)} \gamma_{a}^{p}
\end{aligned}
$$

We first consider each elements on the left side as below:

$$
\sum_{a \in \widehat{A}(p)} t_{a} \pi_{a}^{p}=\sum_{a \in \widehat{A}(p)}\left(\gamma_{a}^{p}-\mu_{a}^{\pi, p}\right) \pi_{a}^{p}=\sum_{a \in \widehat{A}(p)}\left(\gamma_{a}^{p} \pi_{a}^{p}-\mu_{a}^{\pi, p} \pi_{a}^{p}\right)=\sum_{a \in \hat{A}(p)} \gamma_{a}^{p} \pi_{a}^{p}
$$

where the first equality comes from (B.2), the last equality comes from (B.11).

$$
\sum_{a \in \widehat{A}(p)} \bar{t}_{a} \eta_{a}^{p}=\sum_{a \in \widehat{A}(p)}\left(\gamma_{a}^{p}+\mu_{a}^{\eta, p}\right) \eta_{a}^{p}=\sum_{a \in \widehat{A}(p)}\left(\gamma_{a}^{p} \eta_{a}^{p}+\mu_{a}^{\eta, p} \eta_{a}^{p}\right)=\sum_{a \in \widehat{A}(p)} \gamma_{a}^{p} \eta_{a}^{p}
$$

where the first equality comes from (B.3), the last equality comes from (B.12).

$$
\begin{aligned}
& \sum_{a \in A(p)} d_{a} \varpi \kappa_{a}^{p}=\sum_{(i, j)=a \in A(p) \backslash \hat{A}(p)}\left(\beta_{i}^{p}-\beta_{j}^{p}\right) \kappa_{a}^{p}+\sum_{(i, j)=a \in \hat{A}(p)}\left(e \lambda_{a}^{p}+\beta_{i}^{p}-\beta_{j}^{p}\right) \kappa_{a}^{p} \\
= & \sum_{(i, j)=a \in A(p)}\left(\beta_{i}^{p}-\beta_{j}^{p}\right) \kappa_{a}^{p}+\sum_{a \in \hat{A}(p)} e \lambda_{a}^{p} \kappa_{a}^{p} \\
= & \sum_{(i, j)=a \in A(p)}\left(\beta_{i}^{p}-\beta_{j}^{p}\right) \kappa_{a}^{p}+\sum_{a \in \widehat{A}(p)}-\varphi_{a}^{p} \lambda_{a}^{p} \\
= & \sum_{(i, j)=a \in A(p)}\left(\beta_{i}^{p}-\beta_{j}^{p}\right) \kappa_{a}^{p}+\sum_{a \in \hat{A}(p)}-\varphi_{a}^{p}\left(\gamma_{a}^{p}-\mu_{a}^{\varphi, p}\right) \\
= & \sum_{(i, j)=a \in A(p)}\left(\beta_{i}^{p}-\beta_{j}^{p}\right) \kappa_{a}^{p}+\sum_{a \in \hat{A}(p)}\left(-\varphi_{a}^{p} \gamma_{a}^{p}+\varphi_{a}^{p} \mu_{a}^{\varphi, p}\right) \\
= & \sum_{(i, j)=a \in A(p)}\left(\beta_{i}^{p}-\beta_{j}^{p}\right) \kappa_{a}^{p}+\sum_{a \in \hat{A}(p)}-\varphi_{a}^{p} \gamma_{a}^{p}
\end{aligned}
$$

where the first equality comes from (B.5) and (B.6), the third equality comes from (B.10), the fourth equality comes from (B.4), and the last equality is from (B.13).

$$
\begin{aligned}
& \sum_{i \in N(p)} L_{\max } \xi_{i}^{p}=\sum_{i \in N(p)}\left(\beta_{i}^{p}+\mu_{i}^{\xi, p}\right) \xi_{i}^{p}=\sum_{i \in N(p)}\left(\beta_{i}^{p} \xi_{i}^{p}+\mu_{i}^{\xi, p} \xi_{i}^{p}\right) \\
= & \sum_{i \in N(p)} \beta_{i}^{p} \xi_{i}^{p}
\end{aligned}
$$

where the first equality comes from (B.7), and the last equality comes from (B.14). 
$L_{o} \theta_{o(w)}^{p}=\beta_{o(w)}^{p} \theta_{o(w)}^{p}$, due to (B.8).

Therefore, we have

$$
\begin{aligned}
& \sum_{a \in \hat{A}(p)} t_{a}\left(v_{a}\right) \pi_{a}^{p}-\sum_{a \in \hat{A}(p)} \bar{t}_{a} \eta_{a}^{p}-\sum_{a \in A(p)} d_{a} \varpi \kappa_{a}^{p}-\sum_{i \in N(p)} L_{\max } \xi_{i}^{p}+L_{o} \theta_{o(w)}^{p}+\sum_{i \in N(p)} m^{w} \varsigma_{i}^{p} \\
& =\sum_{a \in \widehat{A}(p)} \gamma_{a}^{p} \pi_{a}^{p}-\sum_{a \in \widehat{A}(p)} \gamma_{a}^{p} \eta_{a}^{p}+\sum_{(i, j)=a \in A(p)}\left(-\beta_{i}^{p}+\beta_{j}^{p}\right) \kappa_{a}^{p}+\sum_{a \in \widehat{A}(p)} \varphi_{a}^{p} \gamma_{a}^{p}-\sum_{i \in N(p)} \beta_{i}^{p} \xi_{i}^{p} \\
& +\beta_{o(w)}^{p} \theta_{o(w)}^{p}+\sum_{i \in N(p)} m^{w} s_{i}^{p} \\
& =\sum_{a \in \hat{A}(p)} \gamma_{a}^{p}\left(\pi_{a}^{p}-\eta_{a}^{p}+\varphi_{a}^{p}\right)+\beta_{o(w)}^{p}\left(-\kappa_{o(w) j}^{p}-\xi_{o(w)}^{p}+\theta_{o(w)}^{p}\right)+\beta_{d(w)}^{p}\left(\kappa_{k d(w)}^{p}-\xi_{d(w)}^{p}\right) \\
& +\sum_{i \in N(p) \backslash\{o(w), d(w)\}} \beta_{i}^{p}\left(-\kappa_{i j}^{p}+\kappa_{k i}^{p}-\xi_{i}^{p}\right)+\sum_{i \in N(p)} m^{w} \varsigma_{i}^{p} \\
& =\sum_{a \in \widehat{A}(p)} \gamma_{a}^{p}+\beta_{o(w)}^{p}\left(-\varsigma_{o(w)}^{p}\right)+\beta_{d(w)}^{p}\left(-\varsigma_{d(w)}^{p}\right)+\sum_{i \in N(p) \backslash\{o(w), d(w)\}} \beta_{i}^{p}\left(-\varsigma_{i}^{p}\right)+\sum_{i \in N(p)} m^{w} \varsigma_{i}^{p} \\
& =\sum_{a \in \hat{A}(p)} \gamma_{a}^{p}+\sum_{i \in N(p)}\left(m^{w}-\beta_{i}^{p}\right) \varsigma_{i}^{p} \\
& =\sum_{a \in \widehat{A}(p)} \gamma_{a}^{p}+\sum_{i \in N(p)}\left(-\mu_{i}^{\varsigma, p}\right) \varsigma_{i}^{p} \\
& =\sum_{a \in \widehat{A}(p)} \gamma_{a}^{p}
\end{aligned}
$$

where the third equality comes from (33)-(36), the fifth equality comes from (B.9), and the last equality is from (B.15).

Therefore, the constraint (B.1) can be written as below:

$$
\sum_{a \in A \backslash \hat{A}(p)} t_{a}+\sum_{a \in \hat{A}(p)} \gamma_{a}^{p}-\alpha_{p}-\rho^{w}=0 \quad \forall p \in \hat{P}^{w}, w \in W
$$

As a result, comparing the optimality conditions of NE-VI eliminating $\mu_{a}^{\pi, p}, \mu_{a}^{\eta, p}, \mu_{a}^{\varphi, p}, \mu_{i}^{\xi, p}, \mu_{i}^{\varsigma, p}$, and $\alpha_{p}$, with the network equilibrium conditions (1)-(31) eliminating $\bar{\alpha}_{a}^{p}$, we can find that they are exactly the same except that: $s_{a}^{p}=\gamma_{a}^{p}, \hat{s}_{a}^{p}=\lambda_{a}^{p}, L_{i}^{p}=\beta_{i}^{p}, \bar{\kappa}_{i j}^{p}=$ $-\kappa_{i j}^{p}, \bar{\xi}_{i}^{p}=\xi_{i}^{p}, \bar{\varphi}_{a}^{p}=\varphi_{a}^{p}, \bar{\pi}_{a}^{p}=\pi_{a}^{p}, \bar{\eta}_{a}^{p}=\eta_{a}^{p}, \bar{\theta}_{o(w)}^{p}=-\theta_{o(w)}^{p}, \bar{\beta}_{i}^{p}=\varsigma_{i}^{p}$, and $\mu^{w}=\rho^{w}$. Therefore, NE-VI is equivalent to the network equilibrium conditions. 